CrossMark \& click for updates

Cite this: Mater. Horiz., 2016, 3, 186

Received 4th December 2015, Accepted 11th March 2016

DOI: $10.1039 / \mathrm{c} 5 \mathrm{mh} 00288 \mathrm{e}$

www.rsc.li/materials-horizons

\title{
Recent progress in fabrication techniques of graphene nanoribbons
}

\begin{abstract}
Wentao Xu and Tae-Woo Lee*
Graphene has been the focus of research since its isolation in 2004. However, the lack of a bandgap restricts its application in semiconductor industry in spite of its predicted extremely high carrier mobility ( $>250000 \mathrm{~cm}^{2} \mathrm{~V}^{-1} \mathrm{~s}^{-1}$ ). Theoretical studies predict that a band gap will open in quasi-one-dimensional graphene ribbons, due to quantum confinement and edge effects. Consequently, many approaches to prepare GNRs have been developed. However, applications of GNRs require scalable and easy production with precise control of individual nanostructures. In this paper, we review strategies for fabrication of large-scale GNRs, with a particular focus on methods that control their alignments. The advantages and disadvantages of each method are compared, to suggest development directions in this research field.
\end{abstract}

\section{Introduction}

\subsection{Why graphene nanoribbons are needed}

Graphene was first isolated by exfoliation in 2004 by A. K. Geim and K. S. Novoselov, who were awarded the Nobel Prize in 2010 for this accomplishment. Graphene has since been the topic of

Department of Materials Science and Engineering, Pohang University of Science and Technology (POSTECH), Pohang 790-784, Republic of Korea.

E-mail: twlee@postech.ac.kr, taewlees@gmail.com

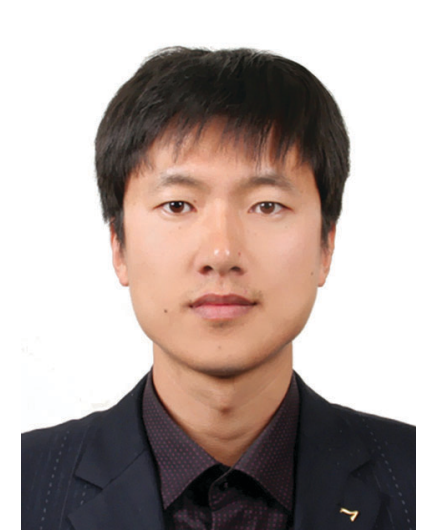

Wentao Xu
Wentao $\mathrm{Xu}$ is a research associate professor in the department of Materials Science and Engineering at Pohang University of Science and Technology (POSTECH), Korea. He received his $P h D$ in Chemical Engineering from POSTECH in February 2012. His research interests include graphene electronics, synapseemulating electronic devices, nonvolatile memory devices and organic field-effect transistors. considerable research because of its superior electronic (theoretically predicted carrier mobility $>250000 \mathrm{~cm}^{2} \mathrm{~V}^{-1} \mathrm{~s}^{-1}$ at room temperature (RT)), chemical and mechanical properties, and high transparency. ${ }^{1-7}$ However, graphene has a zero band gap (Fig. 1), ${ }^{8}$ so its applications in semiconductor technologies are restricted. Several methods to open a band gap in graphene have been developed, including doping, hydrogenation, and fabrication of nanoribbons, nanomeshes and nanorings. ${ }^{9,10}$

Theoretical studies have predicted that confinement of charge carriers in quasi-one-dimensional (Q1D) nanoscale systems will

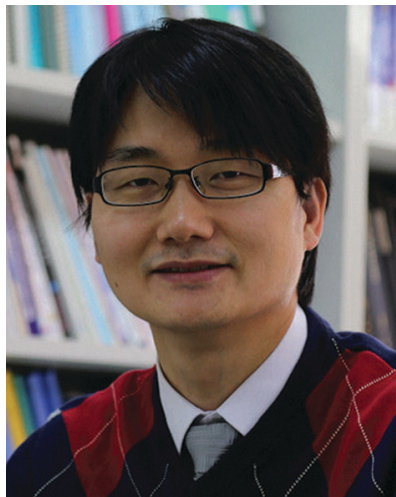

Tae-Woo Lee
Tae-Woo Lee is an associate professor in the department of Materials Science and Engineering at POSTECH, Korea. He received his $P h D$ in Chemical Engineering from Korea Advanced Institute of Science and Technology (KAIST), Korea, in February 2002. He then joined Bell Laboratories, USA, as a postdoctoral researcher in 2002. From September 2003 to August 2008, he worked in the Samsung Advanced Institute of Technology as a member of research staff. He received a prestigious Korea Young Scientist Award from the President of Korea in 2008 and The Scientist of the Month Award from the Ministry of Science, ICT and Future Planning in 2013. His research focuses on printed and organic electronics based on organic and carbon materials for flexible electronics, displays, solid-state lightings, and solar energy conversion devices. 


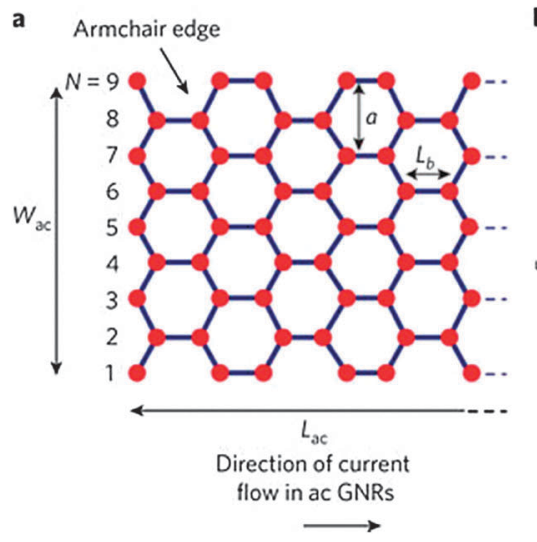

b

Fig. 1 (a) Schematic demonstration of an armchair GNR. (b) Band structure near the $K$ point of (i) bulk graphene, (ii) graphene nanoribbons, (iii) bilayer graphene, and (iv) bilayer graphene in an applied perpendicular electric field. (Reproduced with permission from ref. 8, Copyright 2010, Nature Publishing Group.)
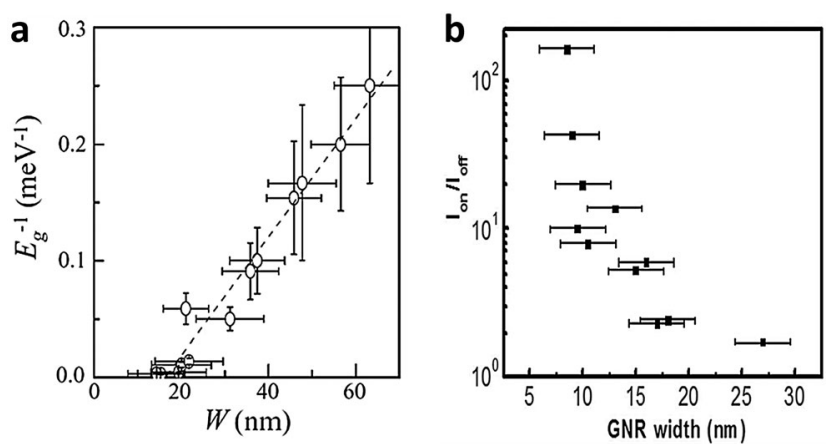

Fig. 2 (a) Reciprocal of bandgap $E_{g}$ as a function of the GNR width $W$. (Reproduced with permission from ref. 18, Copyright 2007, The American Physical Society.) (b) Correlation between on/off current ratio and GNR width. (Reproduced with permission from ref. 12, Copyright 2009, The American Chemical Society.)

open a bandgap,${ }^{11}$ and that the band gap of graphene nanoribbons (GNRs) is inversely proportional to the ribbon width (Fig. 2a). As Q1D materials, GNRs are especially interesting due to their compatibility with nanoelectronics, and their possible applications such as optoelectronics and sensors. In graphene nanoribbon field-effect transistors (GNRFETs), the logarithm of on/off current ratio is proportional to the ribbon width (Fig. 2b); this relationship is regarded as evidence of the opening of a band gap. ${ }^{12}$

\subsection{Basic properties of GNRs}

GNRs are usually classified into armchair (A-GNR) and zigzag (Z-GNR) morphologies depending on the edge structures. The characteristics of the two types of GNRs differ. Theoretical studies have predicted that A-NRs and Z-GNRs will have a band gap that is inversely proportional to the ribbon width. ${ }^{13,14}$ However, the studies disagree about whether Z-GNRs can have a direct bandgap. Predictions also suggest that the edge structure will affect the magnetic properties: A-GNRs are expected to be nonmagnetic, but Z-GNRs could have a large magnetoresistance. ${ }^{13,15-17}$

The correlation between the bandgap and the width of a GNR was experimentally proven for the first time by Kim et al. in $2007 .^{18}$
Lateral confinement of charge carriers induced the formation of a bandgap. The bandgap was strongly correlated with the width $W$ of the GNR (Fig. 2a). However, no clear relationship between electrical characteristics and crystallographic orientation was observed in GNRs produced using e-beam lithography. Lithographic approaches usually produce disordered edges, which may influence the electrical properties of GNRs. Anderson localization has been predicted to induce a bandgap in GNRs (both $\mathrm{A}$ and $\mathrm{Z}$ ) that have disordered edges. ${ }^{19,20}$ If the edges of a GNR are not very rough, the bandgap of the GNR can be roughly estimated as $E_{\mathrm{g}} \approx \alpha / W$, where $\alpha$ is a fitted constant. ${ }^{21-23}$

\subsection{Some techniques for GNR fabrication}

Numerous research papers have presented approaches to produce GNRs and to study their electrical and photonic properties for future industrial applications (Table 1). The following section describes some techniques to produce narrow GNRs (Fig. 3).

E-beam lithography. E-beam lithography (EBL) is a sophisticated tool to produce nanoscale patterns. Kim et al. reported the fabrication of GNRs using e-beam lithography in $2007 .{ }^{18}$ Mechanically exfoliated graphene flakes from bulk graphite crystallites were transferred to a $\mathrm{SiO}_{2}$-coated substrate. ${ }^{24}$ Hydrogen silsesquioxane (HSQ) was used as an e-beam resist to form line patterns with width $10 \leq W_{\mathrm{P}} \leq 100 \mathrm{~nm}$ and length $1 \leq L_{\mathrm{P}} \leq 2 \mu \mathrm{m}$ to serve as protective masks. The unprotected regions of graphene were removed using oxygen plasma to leave GNRs with width $14 \leq W_{\mathrm{P}} \leq 100 \mathrm{~nm}$ beneath HSQ. The bandgap of the GNRs was closely related to $W_{\mathrm{P}}$.

Theoretical studies have suggested that the orientation of a GNR may influence its electrical properties. However, no obvious correlations have been observed; this result implies that the edge structure has a stronger influence on the properties of a GNR than does crystallographic orientation.

Other theoretical work has suggested that $E_{\mathrm{g}}$ is influenced by atomic structures on the edges of GNRs. ${ }^{13,15,25-32}$ No obvious dependence of electrical characteristics on crystallographic orientation has been observed; this result indicates that e-beam lithography did not achieve precise control of the edges of the GNRs. 
Table 1 Summary of the technical methods and properties of graphene nanoribbons

\begin{tabular}{|c|c|c|c|c|c|c|}
\hline Methods & GNR width & Edge & Mobility $\left(\mathrm{cm}^{2} \mathrm{~V}^{-1} \mathrm{~s}^{-1}\right)$ & $I_{\mathrm{ON}} / I_{\mathrm{OFF}}{ }^{a}$ & $E_{\mathrm{g}}(\mathrm{meV})$ & Ref. \\
\hline \multirow[t]{5}{*}{ E-beam litho. } & $14-100 \mathrm{~nm}$ & N/A & N/A & $>10^{3}(49 \mathrm{~nm}, 1.7 \mathrm{~K})$ & $200(15 \mathrm{~nm})$ & 19 \\
\hline & $20 \mathrm{~nm}$ & $\mathrm{~N} / \mathrm{A}$ & N/A & $>100(4 \mathrm{~K})$ & $\sim 30$ & 33 \\
\hline & $12 \mathrm{~nm}$ & N/A & $\mathrm{N} / \mathrm{A}$ & $10(300 \mathrm{~K}) ; 10^{6}(4 \mathrm{~K})$ & 100 & 34 \\
\hline & $5 \mathrm{~nm}$ & (RMS) & $(10 \mathrm{~nm})$ & & 300 & \\
\hline & $5 \mathrm{~nm}$ & N/A & N/A & $47(\mathrm{RT}) ; 10^{5}(5.4 \mathrm{~K})$ & 145 & 36 \\
\hline Dip-pen lithography & $25 \mathrm{~nm}$ & N/A & 2740 (h) 3150 (e) & N/A & N/A & 46 \\
\hline \multirow[t]{3}{*}{ STM } & $10 \mathrm{~nm}$ & Armchair or zigzag & N/A & N/A & 0.18 & 47 \\
\hline & $2.5 \mathrm{~nm}$ & & & & 1.2 & \\
\hline & $1.7 \mathrm{~nm}$ & & & & & \\
\hline \multirow{12}{*}{$\begin{array}{l}\text { Chemical synthesis in } \\
\text { solution or on } \\
\text { crystalline terraces }\end{array}$} & 7 units & Armchair & N/A & N/A & 2300 & 54 \\
\hline & 7 units & Armchair & N/A & $\mathrm{N} / \mathrm{A}$ & $\mathrm{N} / \mathrm{A}$ & 55 \\
\hline & $\sim 2$ & N/A & $\sim 200$ & $>10^{6}$ & N/A & 56 \\
\hline & 13, 7 units & Armchair & N/A & N/A & $\begin{array}{l}1400 ; 2500 \\
(13,7 \text { units })\end{array}$ & 57 \\
\hline & 1 & Armchair & N/A & N/A & 1300 & 58 \\
\hline & 0.74 & Armchair & $\mathrm{N} / \mathrm{A}$ & $3.63 \times 10^{3}(77 \mathrm{~K})$ & 1250 & 59 \\
\hline & 2 & N/A & N/A & N/A & 1240 & 60 \\
\hline & 6 & Armchair and zigzag & N/A & N/A & 1400 & 61 \\
\hline & $0.69-1.13$ & Cove-type & 150-15000 & N/A & 1880 & 62 \\
\hline & $1.2-3.4$ & Chlorinated & N/A & $\mathrm{N} / \mathrm{A}$ & 1700 & 63 \\
\hline & $1.1-0.7$ & Cove-type & N/A & N/A & 1840 & 64 \\
\hline & $<2 \mathrm{~nm}$ & Zigzag & N/A & N/A & N/A & 65 \\
\hline \multirow[t]{2}{*}{ Lattice oriented } & $<10 \mathrm{~nm}$ & Armchair $<0.5 \mathrm{~nm}$ & N/A & $\sim 100(\max )$ & N/A & 81 \\
\hline & $20-30 \mathrm{~nm}$ & Zigzag & N/A & N/A & N/A & 87 \\
\hline DNA assisted & $<10 \mathrm{~nm}$ & $\mathrm{~N} / \mathrm{A}$ & 0.21 & 100-500 (RT) & $\mathrm{N} / \mathrm{A}$ & 88 \\
\hline \multirow[t]{8}{*}{ Unzipping of CNTs } & $>100 \mathrm{~nm}$ & Oxidized & N/A & $<2$ & N/A & 91 \\
\hline & $22-26 \mathrm{~nm}$ & Oxidized & N/A & N/A & N/A & 92 \\
\hline & $130-250 \mathrm{~nm}$ & Non-oxidized & $\mathrm{N} / \mathrm{A}$ & $\mathrm{N} / \mathrm{A}$ & $\mathrm{N} / \mathrm{A}$ & 93 \\
\hline & $2.8 \mathrm{~nm}$ & Zigzag dominant & 1800 & $<2$ & N/A & 94 \\
\hline & $<10 \mathrm{~nm}$ & Smooth & N/A & $>10(7 \mathrm{~nm})>100(6 \mathrm{~nm})$ & N/A & 95 \\
\hline & $10-20 \mathrm{~nm}$ & N/A & 1500 & $4.5(\mathrm{RT}) ; 10(20 \mathrm{~K})$ & $10-15$ & 96 \\
\hline & $4-26 \mathrm{~nm}$ & Armchair and zigzag & $\mathrm{N} / \mathrm{A}$ & $\mathrm{N} / \mathrm{A}$ & N/A & 97 \\
\hline & $2-8 \mathrm{~nm}$ & Smooth & $\mathrm{N} / \mathrm{A}$ & N/A & N/A & 98 \\
\hline \multirow[t]{4}{*}{ BCP self-assembly } & $9-12 \mathrm{~nm}$ & N/A & $120(\mathrm{e}) 60(\mathrm{~h})$ & $100(100 \mathrm{~K})$ & $\begin{array}{l}58(12 \mathrm{~nm}) \\
8(9 \mathrm{~nm})\end{array}$ & 108 \\
\hline & $\sim 12 \mathrm{~nm}$ & N/A & N/A & $<2$ & N/A & 109 \\
\hline & $\sim 10 \mathrm{~nm}$ & N/A & N/A & N/A & N/A & 110 \\
\hline & $8 \mathrm{~nm}$ & $\sim 2.4 \mathrm{~nm}(3 \sigma)$ & 25 & $13(\mathrm{RT})$ & N/A & 111 \\
\hline Edge-narrowing & Sub-5 nm & $\leq 5 \mathrm{~nm}$ & N/A & $10^{4}(\mathrm{RT})$ & 400 & 113 \\
\hline ALD-on edge & $15 \mathrm{~nm}$ & N/A & $\sim 400(15 \mathrm{~nm})$ & $\sim 4$ & N/A & 118 \\
\hline Meniscus mask & $6.4 \mathrm{~nm}$ & Armchair $^{e}$ & $14\left(\mathrm{~h}^{+}\right)$ & $10(\mathrm{RT}) ; 10^{3}(5 \mathrm{~K})$ & 120 & 119 \\
\hline Barrier-guided & $25 \mathrm{~nm}$ & N/A & 215 & $\sim 6$ & 15 & 120 \\
\hline
\end{tabular}


Table 1 (continued)

\begin{tabular}{llllll}
\hline Methods & GNR width & Edge & Mobility $\left(\mathrm{cm}^{2} \mathrm{~V}^{-1} \mathrm{~s}^{-1}\right)$ & $I_{\mathrm{ON}} / I_{\mathrm{OFF}}{ }^{a}$ & \\
\hline i-NW lithography & $8 \mathrm{~nm}$ & N/A & & 160 & $E_{\mathrm{g}}(\mathrm{meV})$ \\
& $50-100 \mathrm{~nm}$ & N/A & 1200 & $13-51$ & 12 \\
& $15 \mathrm{~nm}$ & N/A & 1300 & 70 & 130 \\
& $10 \mathrm{~nm}$ & N/A & & $3000^{f}$ & 131 \\
& $40 \mathrm{~nm}$ & N/A & & & 134 \\
& $6 \mathrm{~nm}$ & N/A & & 70 & 138 \\
e-NW lithography & $9 \mathrm{~nm}$ & N/A & 300 & 143
\end{tabular}

${ }^{a} I_{\mathrm{ON}} / I_{\mathrm{OFF}}$ at RT if not specifically noted. ${ }^{b}$ Synthesized from perianthracene. ${ }^{c}$ Synthesized from perinaphthalene. ${ }^{d}$ Synthesized from peritetracene. ${ }^{e}$ Armchair edges produced in Ar-RIE. ${ }^{f}$ An $I_{\mathrm{ON}} / I_{\mathrm{OFF}}$ of $\sim 3000$ was achieved in a vertical electrical field for a bilayer GNR.

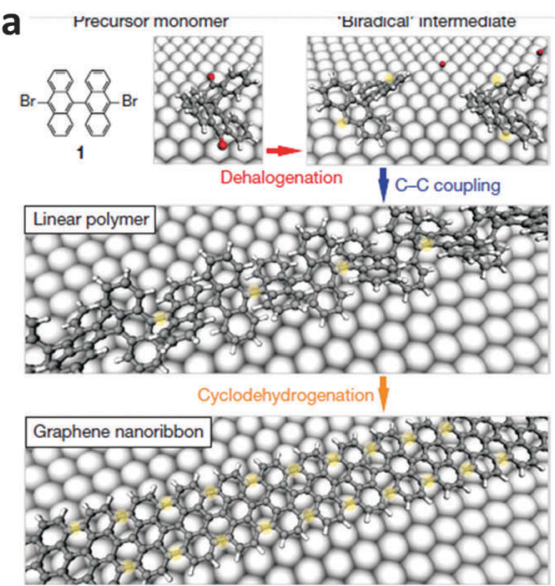

b

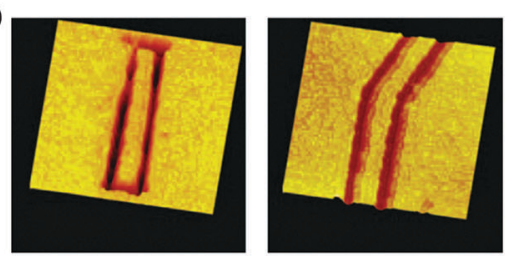

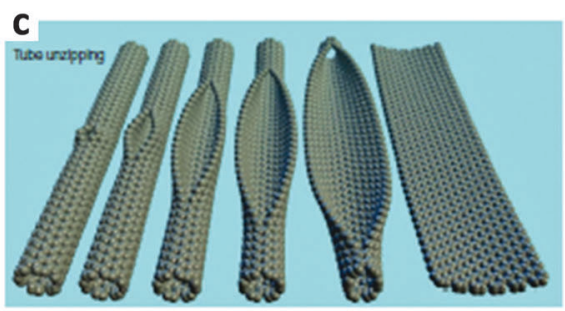

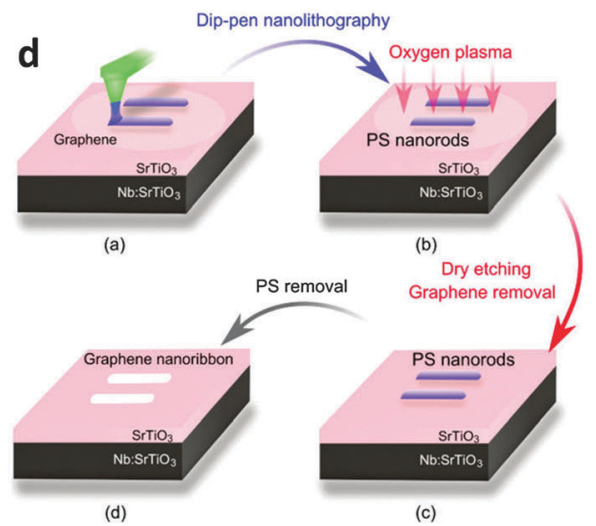

Fig. 3 Some techniques to fabricate GNRs. (a) Chemical synthesis from selected monomers. (Reproduced with permission from ref. 51, Copyright 2010, Nature Publishing Group). (b) STM lithography. (Reproduced with permission from ref. 47, Copyright 2008, Nature Publishing Group). (c) Unzipping of GNTs. (Reproduced with permission from ref. 91, Copyright 2009, Nature Publishing Group). (d) Dip-pen nanolithography. (Reproduced with permission from ref. 46, Copyright 2011, Nature Publishing Group.)

Other groups also used e-beam lithography to fabricate GNRs. ${ }^{33-36}$ Hwang et al. used e-beam lithography ${ }^{34}$ with methylisobutylketone (MIBK)-diluted HSQ as an e-beam resist and a CVD-grown graphene sheet that was transferred to a $90 \mathrm{~nm}$ $\mathrm{SiO}_{2}$-coated $\mathrm{p}+\mathrm{Si}$ wafer for patterning; they produced GNRs with $W=12 \mathrm{~nm}$ (Fig. 4a). The $12 \mathrm{~nm}$-wide GNR was connected to a graphene contact pad through a linear lithographic flare to achieve a continuous change of the GNR bandgap to the zero bandgap of bulk graphene electrodes (Fig. 4b). The RMS roughness of the GNRs was $\sim 0.35 \mathrm{~nm}$. Ambipolar charge transport was observed in the device. GNRFETs had an on/off current ratio $I_{\mathrm{ON}} / I_{\mathrm{OFF}}$ of $\sim 10$ at RT and this was as high as $10^{6}$ at $4 \mathrm{~K}$ (Fig. 4c). A bandgap of $\sim 100 \mathrm{meV}$ was calculated by using a differential conductance method to evaluate the GNRFETs (Fig. 4d).

Although EBL can produce GNRs with precise positioning, it is a high-vacuum, time-consuming serial process, so it is not amenable to scale-up for industrial-scale production. EBL also has resolution limitation that hinders the production of GNRs with width $<10 \mathrm{~nm}$, which are usually required for the formation of a sufficiently large band gap for RT applications. Another drawback is that the etching process causes damage, so atomically smooth level edges are not achievable, and this process also produces numerous dangling bonds and chemical functional groups on the edges of GNRs; as a result, their electrical properties are degraded. Another drawback is that the resist leaves residue that is difficult to remove completely.

Helium ion beam lithography. Helium ion beam lithography (HIBL) $)^{37,38}$ achieves a small spot size and a small scattering length, ${ }^{39}$ and therefore a finer resolution than EBL. This technique can cut graphene with $\sim 10 \mathrm{~nm}$ feature size. ${ }^{40,41}$ Abbas et al. reported the successful use of HIBL to pattern CVD-grown monolayer graphene into high density GNR arrays. This process achieved precise control of $W$ of GNRs and the distance between them, with $W$ of $5 \sim 6 \mathrm{~nm}$ possible. ${ }^{42} \mathrm{~A}$ high aspect ratio (AR) of 

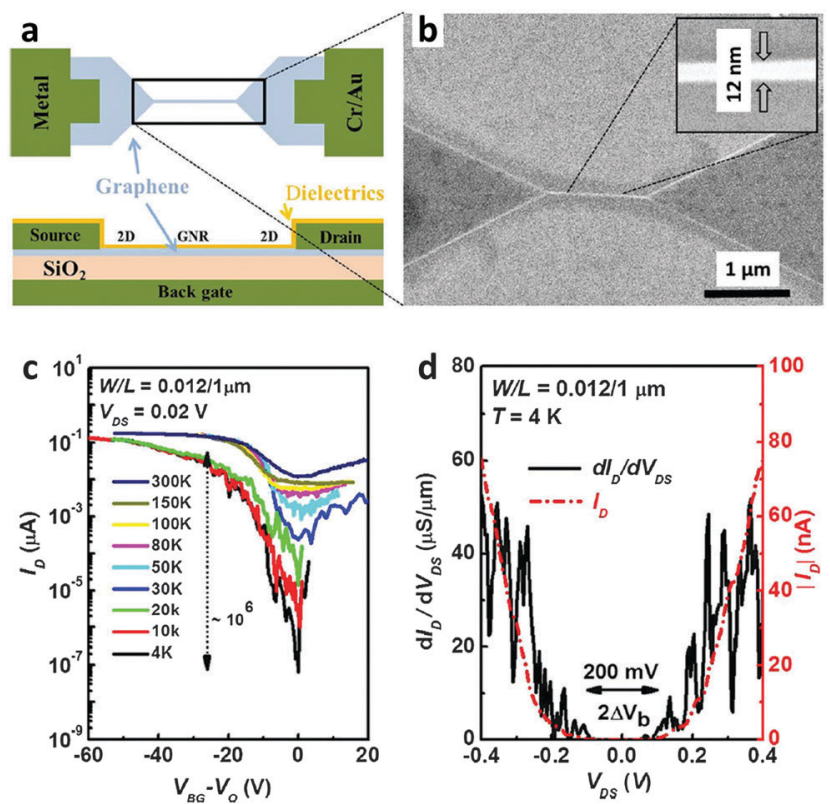

Fig. 4 E-beam lithography to produce graphene nanoribbons (GNRs) (a) Schematic illustration of the configuration of a GNRFET. (b) SEM image of a GNR (inset: magnified view of the nanoribbon with a width of $12 \mathrm{~nm}$ ). (c) Transfer curves of the $12 \mathrm{~nm}$ wide GNRFET at various temperatures. (d) Differential and absolute drain current vs. drain voltage $\left(V_{\mathrm{g}} \sim 50.5 \mathrm{~V}\right)$ (Reproduced with permission from ref. 34, Copyright 2007, AIP Publishing.)

$\sim 400$ was achieved (i.e., $2000 \mathrm{~nm} L \times 5 \mathrm{~nm} \mathrm{~W}$ ). The G-band to D-band ratio $I_{\mathrm{G}} / I_{\mathrm{D}}$ of $\sim 8.39 / W$ in the Raman spectra of GNRs with $W<15 \mathrm{~nm}$ was similar to the $I_{\mathrm{G}} / I_{\mathrm{D}}$ of GNRs produced using EBL; this result means that the two techniques induce similar levels of defects. Multichannel GNRFETs were fabricated using the densely packed GNRs as active channels, which are GNR arrays with $6 \mathrm{~nm}$ half-pitch and $2 \mu \mathrm{m} L$. The minimum conductance decreased and $I_{\mathrm{ON}} / I_{\mathrm{OFF}}$ increased as temperature decreased from RT to $77 \mathrm{~K}$, and the opened band gap was estimated to be $>88 \mathrm{meV}$.

The ability of HIBL to precisely control $W$ down to 5-6 nm revealed on-current degradation as the feature size of GNRs was reduced. Compared to a bulk graphene sheet, the current density was decreased to $\sim 1 / 2$ in a $15 \mathrm{~nm}$-wide GNR and to $\sim 1 / 5000$ in a $6 \mathrm{~nm}$-wide GNR. ${ }^{42}$ In a separate study, the carrier mobility of a $15 \mathrm{~nm}$-wide GNR was $1 / 15$ th that of a $1000 \mathrm{~nm}$-wide GNR. ${ }^{43}$ The degradation of carrier mobility can be attributed to several causes: (1) the edge scattering effect that is inversely proportional to $W$; (2) dangling bonds as surface defect states that form when Si-O bonds break during etching; (3) increased effective electron and hole masses as GNRs become narrow; ${ }^{42,44}$ (4) charge traps produced in $\mathrm{SiO}_{2}$ during etching.

Dip-pen nanolithography. Dip-pen nanolithography (DPN) can draw nanowires and nanorods directly and with precise control. ${ }^{45}$ Shin et al. used polystyrene (PS) solution in toluene as ink and a $\mathrm{S}_{3} \mathrm{~N}_{4}$ DPN tip with radius $<10 \mathrm{~nm}$ to draw PS nanorods on top of a graphene sheet. ${ }^{46}$ The PS nanorods had width $30 \leq W \leq 150 \mathrm{~nm}$. After thermal drying under vacuum, the samples were exposed to oxygen plasma to remove the unprotected area of the graphene sheet. The PS nanorods were finally removed by successive sonication in organic solvents and distilled water. DPN-produced GNRs had a $W$ as low as $25 \mathrm{~nm}$, with an electron mobility of $\sim 3150 \mathrm{~cm}^{2} \mathrm{~V}^{-1} \mathrm{~s}^{-1}$ and a hole mobility of $\sim 2740 \mathrm{~cm}^{2} \mathrm{~V}^{-1} \mathrm{~s}^{-1}$.

Scanning tunneling microscopy lithography ${ }^{47}$. Scanning tunneling microscopy (STM) lithography has atomic-level precision of manipulation, so it can precisely control the edge types during GNR production. Although it is a very powerful tool for the scientific study of the unique properties of GNRs, mass production would be too expensive, and scalable production at the chip level is not envisioned.

Although the above techniques can produce individual GNRs with $W<10 \mathrm{~nm}$, additional requirements must be satisfied before industrial applications are practical. These requirements include alignment ability, uniform control over $W$, a high AR, and edge roughness. The development of scalable approaches to synthesize graphene has enabled fabrication of GNR circuits on a large area; examples include epitaxial graphene sheets on the $\mathrm{SiC}$ surface, ${ }^{48,49}$ and CVD growth on catalytic metal surfaces. ${ }^{6,50}$

Previous review papers about GNRs have summarized the fabrication techniques and the properties of GNRs. However, for real application, fabrication and alignment of GNRs on a large area is essential. The main focus of this paper is to review methods for large-scale fabrication of GNRs, especially those that have shown potential for fabrication of GNRs on a large area with individual control of position and alignment. At least three criteria of GNR fabrication are required for practical application: (1) fabrication of GNRs with $W<10 \mathrm{~nm}$, (2) direct integration to insulating or semiconducting interfaces, and (3) mass production of GNRs with individually controlled position and alignment. Efforts have been made to overcome these challenges.

\section{Current techniques that show potential for large-scale fabrication of GNRs}

Some approaches have shown potential for large-scale fabrication of GNRs with controlled alignment (Fig. 5 and Table 1), which will be discussed in the following sections.

\subsection{Bottom-up organic synthesis method in solution}

GNRs can be chemically synthesized from various monomers. ${ }^{21,51-69}$ The $W$ and edge types (i.e., A or Z) can be defined with atomic precision by designing the structure of precursor monomers. Chemically derived GNRs usually have uniform edges. A very narrow (down to 7 C-atoms wide) $W$ can be obtained with good control. The first graphite nanoribbons were synthesized in solution. ${ }^{67}$ The synthesis is generally based on solution-mediated cyclodehydrogenation and "planarization" of three-dimensional polyphenylene precursors. The polyphenylene precursors are designed and synthesized from small organic molecules, and provide the ability to fabricate GNRs with different widths and edge structures. ${ }^{68}$ Recently, GNRs with $L>600 \mathrm{~nm}$ have been 


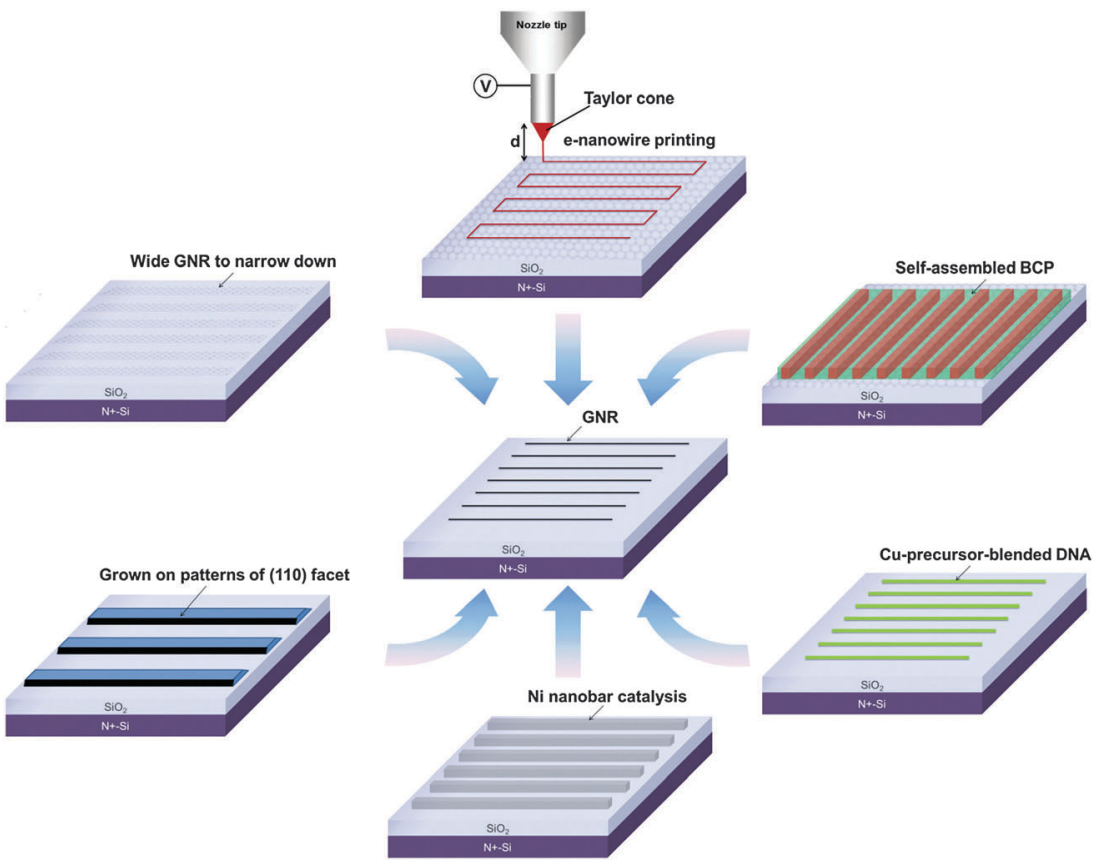

Fig. 5 Demonstration of various techniques for the mass production of GNR arrays.

produced using this bottom-up organic synthesis in solution, ${ }^{62}$ and large-scale solution synthesis of narrow GNRs has also been achieved using this method. ${ }^{58}$ The bottom-up organic synthesis method in solution has been well developed and serves as an indispensable tool to create structurally defined GNRs. In contrast to the "top-down" methods, "bottom-up" organic synthesis in solution provides structurally well-defined GNRs with uniform structures. However, the synthesized GNRs must be transferred to suitable substrates with precise position control; this requirement is a difficult challenge.

Atomically controlled substitutional doping of GNRs can also be achieved using chemical synthesis in solution. Desired elements for either $\mathrm{p}$ - or $\mathrm{n}$-doping can be designed in the monomer for the GNR synthesis, so that these elements are incorporated into the synthesized GNRs. ${ }^{69}$ Although atomically controlled doping of GNRs on a large-scale is difficult at this time, this method provides a practical way to achieve an efficient band gap for GNRs, and could direct future development of this research field.

\subsection{Templated bottom-up growth}

Bottom-up growth avoids etching over GNR edges, and thereby maintains physically smooth and chemically clean edges. This process yields high-quality GNRs, which usually possess higher charge-carrier mobility than that achieved using top-down approaches. The critical step is to obtain pre-patterned catalytic nano-patterns; this goal can be realized by using catalytic edges of thin metal films, or by using other techniques.

Early attempts at bottom-up growth of GNRs used Pd nanowires to catalyze the growth of GNRs as surroundings. ${ }^{70}$ The Pd NWs can then be removed to achieve edge-closed GNRs, or if the top-layer graphene ribbons are etched away by oxygen plasma before the removal of Pd nanowires, edge-open graphene ribbons can be achieved. However, the alignment of individually addressable i-NWs is still a difficult challenge. This technique is very useful for scientific research, but is not suitable for massproduction of addressable GNRs on a large area.

2.2.1. Scalable template growth of graphene nanoribbons on SiC. $\mathrm{SiC}$ is an attractive substrate for epitaxial graphene growth by thermal decomposition. ${ }^{71-73}$ However, the morphology of epitaxial graphene is strongly affected by the underlying $\mathrm{SiC}$ facets. Under some graphene growth conditions, ${ }^{50,72-74}$ terrace structures sometimes appear in the miscut angle of the $\mathrm{SiC}$ wafer. Although the steps were regarded as problematic, ${ }^{75}$ a continuous graphene lattice was observed on them.

Sprinkle et al. exploited this phenomenon to design an approach for bottom-up growth of GNRs (Fig. 6a). ${ }^{76}$ Nickel lines were first fabricated on a flat SiC surface by photolithography followed by fluorine RIE. The edge line patterns of the Ni mask were then transferred to the underlying SiC. This very easy process controlled the etch depth accurately on a nanoscale. The lithographic depth determined the final width of GNRs (Fig. 6a): a $20 \mathrm{~nm}$ lithographic depth yielded $40 \mathrm{~nm}$-wide GNRs, which could be further narrowed by decreasing the lithographic depth. The crystal was heated at $1200-1300{ }^{\circ} \mathrm{C}$ in vacuum after removal of the Ni mask to form a SiC step flow. The abrupt step relaxed into a (110) facet. The temperature was increased to $1450{ }^{\circ} \mathrm{C}$ after $1.5 \mathrm{~min}$ to induce selective growth of GNRs on this nanofacet. ${ }^{71-73}$

Careful control of the temperature, time and atmosphere during CVD growth induces selective growth of GNRs on the facets (Fig. 6b-d). Raman mapping over a $100 \mathrm{~nm} \mathrm{SiC} \mathrm{step} \mathrm{and}$ adjacent facets confirmed the existence of graphene at the step edges by showing strong $2 \mathrm{D}$ bands $\left(2700 \mathrm{~cm}^{-1}\right)$ there, but 


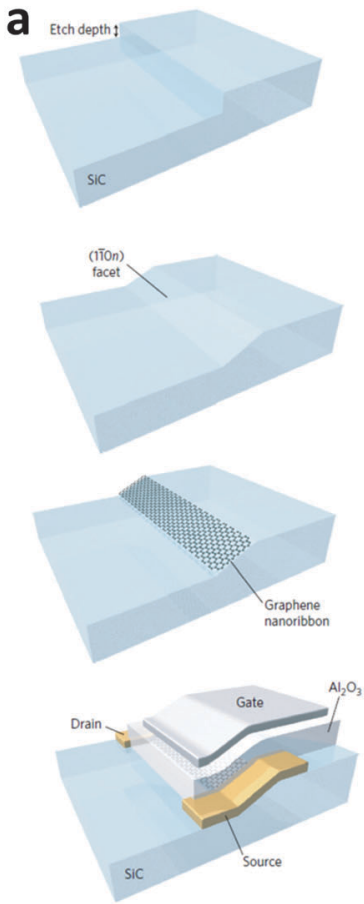

b

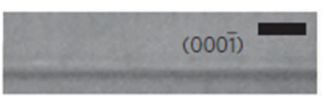

C

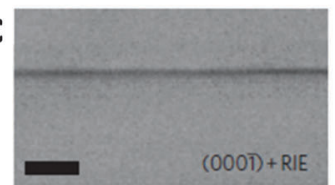

d
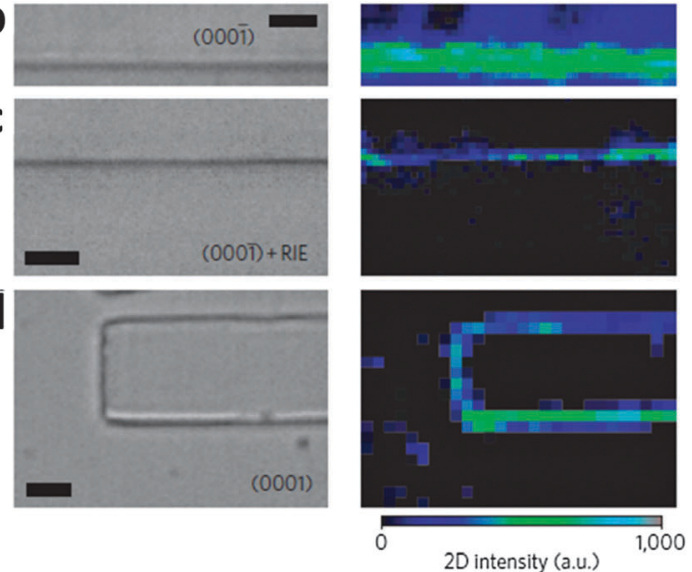

e

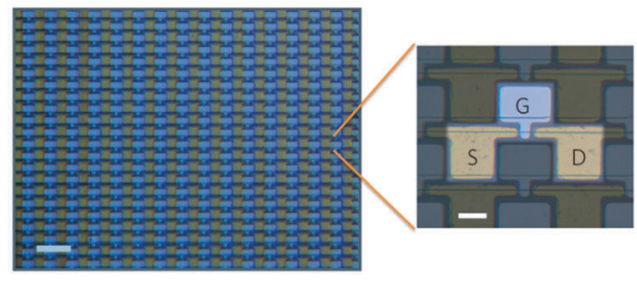

Fig. 6 (a) Fabrication of a nanometer-wide step on $\mathrm{SiC}$ and growth of GNRs on it, and the fabrication of a top-gated GNRFET. Optical micrographs and Raman mapping images of (b) a $100 \mathrm{~nm}$-wide step of the (000-1) facet after graphene growth, (c) the (000-1) facet after graphene growth and oxygen plasma exposure, and (d) selective growth on SiC without post-treatments (scale bar for left images in (b), (c), and (d): $2 \mu \mathrm{m}$ ). (e) Optical micrograph of GNRFET arrays; scale bar: $100 \mu \mathrm{m}$ (left) and $20 \mu \mathrm{m}$ (right). (Reproduced with permission from ref. 76, Copyright 2010, Nature Publishing Group.)

found almost none on the horizontal surfaces. Raman spectroscopy, HR-TEM and EFM confirmed the growth of $40 \mathrm{~nm}$ GNRs at expected positions. The GNRs exhibited the quantum confinement effect at $4 \mathrm{~K}$. Electronic devices based on these GNRs showed an $\mathrm{RT} I_{\mathrm{ON}} / I_{\mathrm{OFF}}$ of $\sim 10$ and a charge carrier mobility of $\sim 2700 \mathrm{~cm}^{2} \mathrm{~V}^{-1} \mathrm{~s}^{-1}$. High-density transistor arrays were fabricated (Fig. 6e). The technique could be further refined by downscaling GNR width, or by selecting different gate dielectrics and crystallographic facets. The bottom-up growth avoids the generation of side defects during cutting by plasma. The GNRs show metallic behavior at relatively high temperature, but quantum confinement at $4 \mathrm{~K}$. Reduction of the width to $\sim 40 \mathrm{~nm}$ yields a bandgap at RT. ${ }^{77}$

\subsubsection{Direct-growth on nickel nanostructures}

2.2.2.1. Growth on nickel nanobars. Ni is a well-known catalytic metal substrate for multi-layer graphene growth. Therefore, $\mathrm{Ni}$ nanostructures should be evaluated as a bed for GNR growth. Kato et al. reported direct growth of GNRs from pre-patterned $\mathrm{Ni}$ nanostructures (Fig. 7a). ${ }^{11}$ In this approach, Ni nanobars with width $50-100 \mathrm{~nm}$, height $35-85 \mathrm{~nm}$ and length $200-5000 \mathrm{~nm}$ were pre-patterned using conventional e-beam lithography and then connected to two $\mathrm{Ni}$ electrodes as the source and drain. Rapid-heating plasma CVD (RH-PCVD) was then applied to these nanostructures, and the patterns were directly converted to GNRs. The sacrificial Ni nanobars were partially or fully eliminated during the growth of GNRs. The keys to this direct conversion process are rapid heating, abundant supply of hydrocarbon sources, and relatively thin $\mathrm{Ni}$ nanostructures. During growth, the narrowest $\mathrm{Ni}$ structures were volatilized to leave GNRs in the channel region, but the Ni electrodes remained. Due to elimination of the Ni structures, the GNRs are usually $\sim 23 \mathrm{~nm}$ narrower than their sacrificial catalytic nanostructures (Fig. 7b). The conversion efficiency was $45-70 \%$. The large height of $\sim 60 \mathrm{~nm}$ suggested a suspended structure of the GNRs. TEM revealed clear graphitic lattice structures with $0.34 \mathrm{~nm}$ spacing and a multilayer graphene sheet with 5-10 layers of graphene. The bottom-up growth avoids lithographic approaches, so it produced GNRs with relatively flat and smooth surfaces and edges. Complex architectures such as multichannel structures and radical line structures are achievable by designing the patterns of the Ni nanostructures. During RH-PCVD, only the narrowest parts of $\mathrm{Ni}$ were converted to GNRs; this trait can be used to ensure that GNRs grow only at specific points in a pre-constructed structure.

Although the site and alignment of GNRs can be controlled, this process still has the limitation that patterning of the $\mathrm{Ni}$ nanostructures requires e-beam lithography, so the problems involved with a serial, time-consuming, and high cost process remain. Due to the resolution limitation in patterning $\mathrm{Ni}$ nanostructures, the narrowest GNR width was $\sim 23 \mathrm{~nm}$.

2.2.2.2. Growth on the edge of nickel thin films. Controlling the thickness of a catalytic metal thin film is probably much easier than laterally confining the width of a nanostructure, because a nanoscale thickness of the film can be easily obtained during the deposition process. This is a possible solution to avoid the e-beam lithography approach to achieve a nanostructured Ni catalytic layer (Fig. 8a). ${ }^{78}$ 


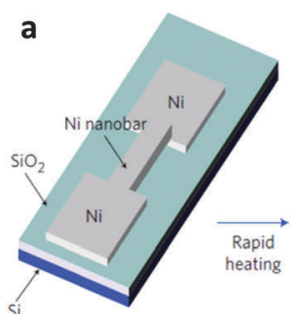

b

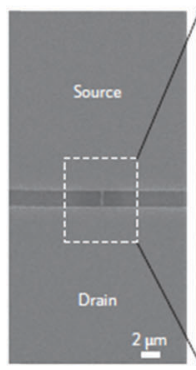

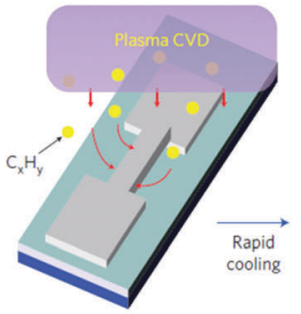
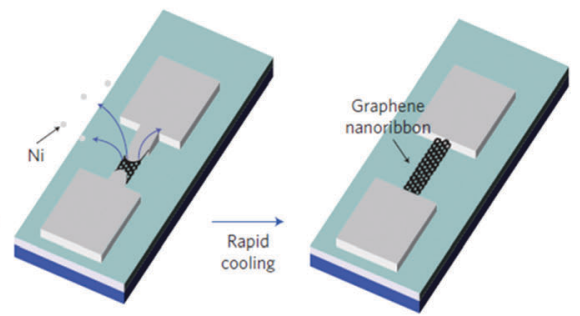

C

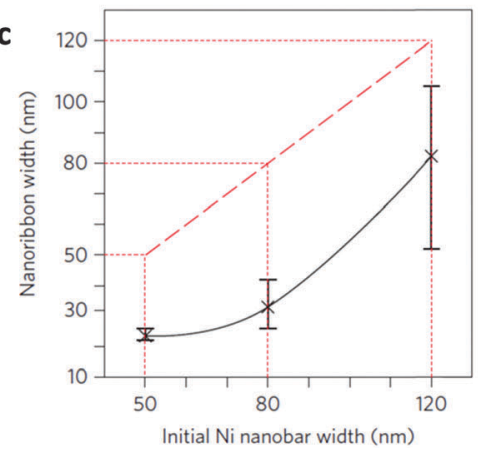

Fig. 7 (a) Schematic of the site- and alignment-controllable fabrication of GNR arrays from nickel nanobars. SEM images of (b) a nickel nanobar and a GNR. (c) Correlations between nanoribbon width and the initial width of the nickel nanobar. (Reproduced with permission from ref. 11, Copyright 2012, Nature Publishing Group.)

A $20 \mathrm{~nm} \mathrm{Ni}$ thin layer is first deposited on a $\mathrm{SiO}_{2}$-coated highly doped silicon wafer and then coated with $75 \mathrm{~nm} \mathrm{Al}_{2} \mathrm{O}_{3}$ as a protective layer. The double layer thin film is then patterned to leave laterally exposed nanoscale $\mathrm{Ni}$ edges with desired patterns. Vertical GNRs with height $\sim 20 \mathrm{~nm}$ were achieved (Fig. 8b). A planar Ni nanostructure is difficult to pattern, but the thickness of the Ni layer can be easily controlled during the deposition process, if an inert protective layer is applied to cover the Ni layer. This is a method to grow vertical GNRs.

2.2.3. Direct growth on other substrates. CVD can be used to grow continuous graphene sheets on a Ge surface ${ }^{79}$ and on the facets of $\mathrm{Ge}(001), \mathrm{Ge}(110)$ and $\mathrm{Ge}(111) .{ }^{80}$ However, the efforts toward graphene growth on the Ge surface have mainly focused on sheet-like graphene growth. Most recently, Jacobberger et al. reported the direct synthesis of GNRs on Ge(001) facets by controlling the growth dynamics (Fig. 9). ${ }^{81}$ These GNRs have $W<10 \mathrm{~nm}$, controllable crystallography, and smooth armchair edges. The orientations of the GNRs depended on the underlying surficial crystal structures. The bottom-up grown GNRs have controllable $W, L$ and AR.

Anisotropic growth of GNRs was achieved on Ge(001) by controlling CVD conditions such as temperature, fractions of $\mathrm{CH}_{4}$ and $\mathrm{H}_{2}$ and growth time. The key to this anisotropic growth is to use growth with a very restricted rate $<5 \mathrm{~nm} \mathrm{~h}^{-1}$. Under optimized conditions, the separated GNRs could be allowed to grow preferentially for $>18 \mathrm{~h}$, whereas thin film growth on $\mathrm{Ge}(001)$ was completed in $<100 \mathrm{~min}^{79}$

The anisotropic growth was due to selective attachment of hydrocarbon intermediate products to short edges rather than to the long ribbon edges. As a consequence, the GNRs grew preferentially in the length direction, and therefore elongated instead of widening. During the growth process, the energy barrier for the intermediate products to attach to the long ribbon edges was determined by the relative positions of GNR
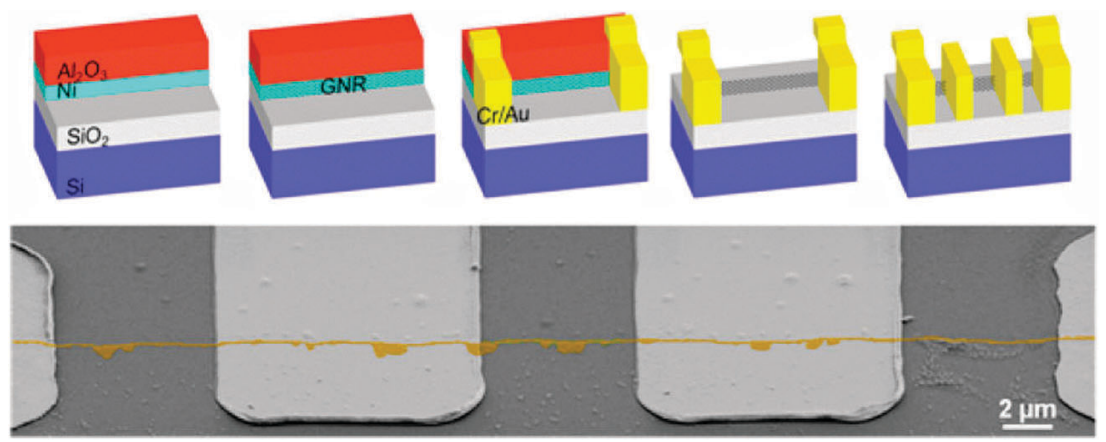

Fig. 8 (a) Schematic of vertical GNR growth on the edges of thin Ni layers with an $\mathrm{Al}_{2} \mathrm{O}_{3}$ passivative layer on top and a GNRFET. (b) SEM image of GNRs with electrodes. (Reproduced with permission from ref. 78, Copyright 2012, The American Chemical Society.) 
a

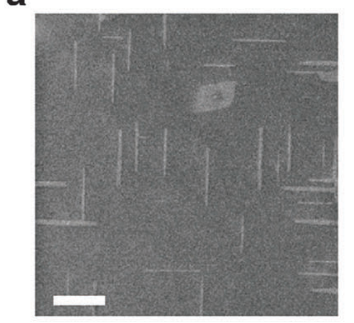

b

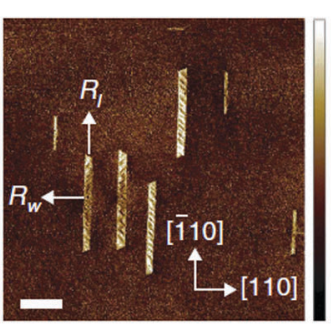

c

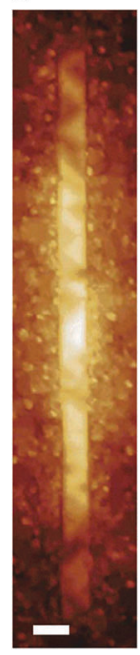

d

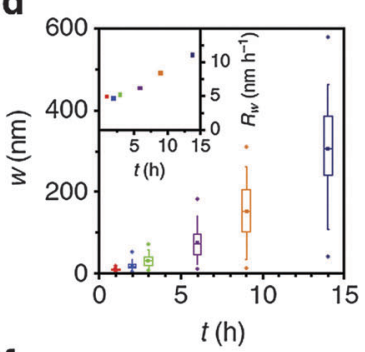

f

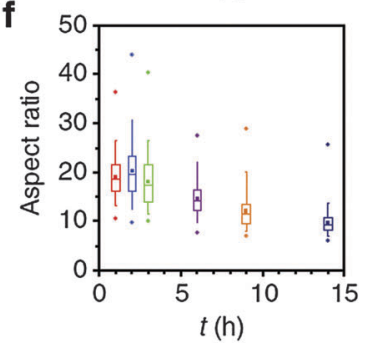

e

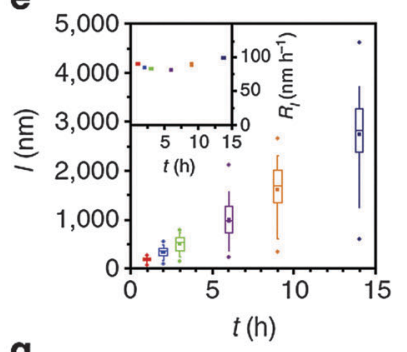

g

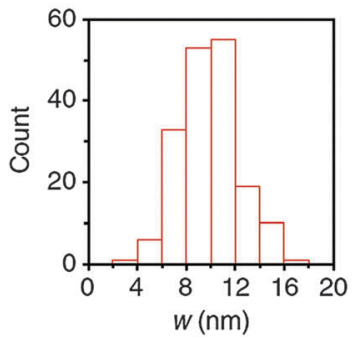

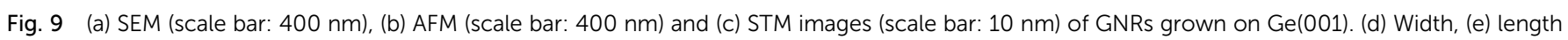

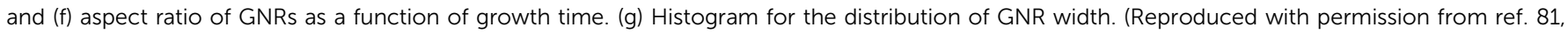
Copyright 2015, Nature Publishing Group.)

edges to Ge steps; this process is similar to the formation of polygonal graphene crystals ${ }^{82}$ and of Q1D structures on zincblende semiconductor (001) facets. ${ }^{83-85}$ However, the barrier for the attachment of intermediate products decreased once GNRs overgrew the Ge steps; furthermore, $W$ increased rapidly when it exceeded $30 \mathrm{~nm}$. The subsequent rapid growth was due to the GNRs exceeding the steps on the Ge surface and thereby overcoming the attachment barrier.

Direct, definable growth provides precise control over GNR growth, and can produce relatively pristine GNR/substrate interfaces. This type of GNR has $W<10 \mathrm{~nm}$, a high AR and very smooth armchair edges. These characteristics indicate that these techniques can be adapted to realize mass production and high yield to directly integrate GNRs into conventional semiconducting wafer platforms. ${ }^{73,86}$ Similar approaches that use surficial lattice structures to orient the growth of GNRs have also been reported. ${ }^{87}$

2.2.4. Direct growth from a DNA template. Bao's group reported the use of CVD to grow graphitic nanoribbons directly from a DNA template. ${ }^{88}$ DNA was selected to form a template because it can be easily stretched by molecular combing. The aligned DNA was useful in the production of metal NWs by reduction of bound metal salts. ${ }^{89}$ Double-stranded lambda DNAs were spin-coated from solution to form aligned patterns and then immersed in $0.1 \mathrm{M} \mathrm{Cu}\left(\mathrm{NO}_{3}\right)_{2}$ aqueous solution. $\mathrm{Cu}^{2+}$ was loaded onto the DNA due to the ability of $\mathrm{Cu}^{2+}$ to catalyze single-layer graphene growth. The wafer was thermally dried and then subjected to CVD. Continuous GNRs were observed over the $5 \mathrm{~mm}$ scan (Fig. 10). Due to the coexistence of $\mathrm{sp}^{2}$ and $\mathrm{sp}^{3}$ characters in the nanoribbons, they were named graphitic nanoribbons. To evaluate the possible effects of defects that were not detectable using AFM, electrical conductivity was measured. The estimated width of the graphitic NRs was $4.9-10 \mathrm{~nm}$. Various patterns can be achieved by altering the alignment of DNAs. Crossbar-architectured graphitic NRs from two perpendicularly aligned DNAs were also fabricated to demonstrate the versatility of the technique to align graphitic NRs.

\subsubsection{Growth from electrospun polymer/catalyst compo-} site nanofibers. Liu et al. reported scalable synthesis of highly graphitized GNRs from polymer/catalyst composite nanofibers. ${ }^{90}$ Pd-incorporated polymer fibers were electrospun onto a rolling collector, which aligned the direction of the collected composite fibers. The aligned fibers were dry-transferred to desired substrates and then subjected to CVD to be converted to GNRs. The $\mathrm{Pd}(\mathrm{OAc})_{2}$ precursor was reduced to Pd during CVD, which then catalyzed graphitization around the Pd nanostructures. A $30 \mathrm{~nm}-$ wide GNR produced using this process was evaluated in a transistor structure at RT; the device had an $I_{\mathrm{ON}} / I_{\mathrm{OFF}}$ of 14 and a field-effect mobility of $\sim 28 \mathrm{~cm}^{2} \mathrm{~V}^{-1} \mathrm{~s}^{-1}$. These results implied that a bandgap had opened in the graphene nanostructure. Further improvement of the technique is expected by precisely controlling the diameter of the electrospun nanofibers.

\subsection{Unzipping carbon nanotubes}

Carbon nanotubes (CNTs) have many similarities to graphene, such as the nanoscale width and Q1D structure. A CNT can be unzipped longitudinally to produce a GNR. Single-walled CNTs can be converted to single-layer GNRs, and multi-walled CNTs can be converted to multi-layer GNRs. ${ }^{91-98}$ The unzipping can be achieved using chemical approaches and controllable RIE etching approaches to partially remove some top parts of a CNT to leave a GNR. Wet chemical reactions and dry etching processes have both been used successfully.

Kosynkin et al. reported an oxidation process in solution to cut multiwalled carbon nanotubes (MWCNTs) lengthwise. ${ }^{91}$ This process yielded nearly $100 \%$ GNR structures with high water solubility. Due to the existence of abundant oxygencontaining functional groups on the surface, the conductivity 

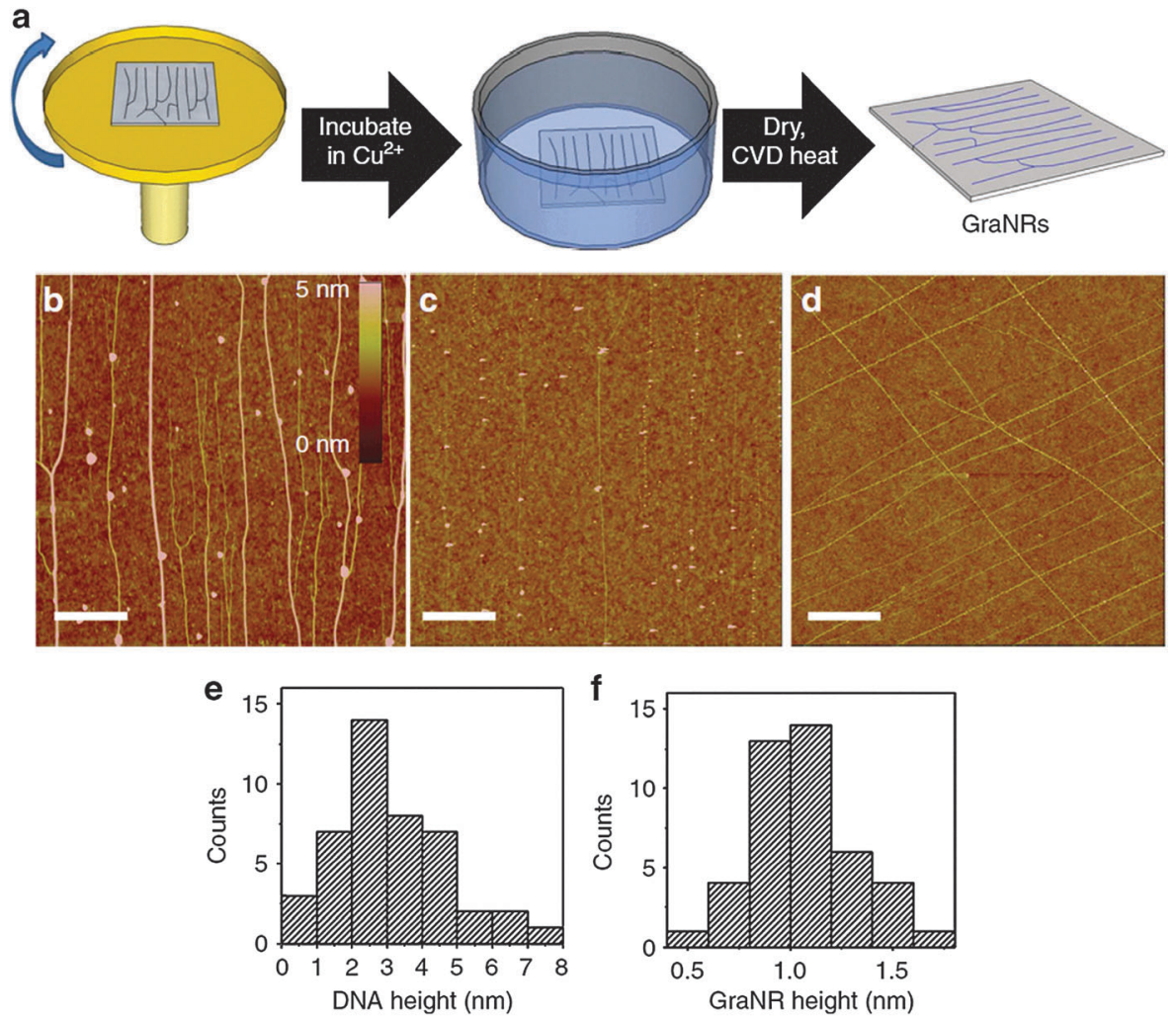

Fig. 10 (a) Schematic of the DNA-template production and its conversion to GNRs. AFM images of (b) stretched DNAs, (c) GNRs and (d) crossshaped GNRs (scale bar: $1 \mu \mathrm{m}$ ). Histograms of the heights of (e) DNAs and (f) GNRs. (Reproduced with permission from ref. 88, Copyright 2013, Nature Publishing Group.)

of the as-produced GNRs was not good. The conductivity can be improved by subsequent chemical reduction by $\mathrm{N}_{2} \mathrm{H}_{4}$ or annealing in $\mathrm{H}_{2}$.

Liao et al. reported a gas-phase plasma etching process to convert masked CNTs to GNRs. ${ }^{95}$ High-quality, narrow GNRs were produced using the approach. ${ }^{96}$ The same group then suggested a two-step process to unzip nanotubes for mass production of high-quality GNRs. Mild calcination at $500{ }^{\circ} \mathrm{C}$ in air induced oxidation of pre-existing defects to produce pits on the side walls of CNTs, and then sonication in organic solvents effectively enlarged the pits and unzipped the CNTs to form GNRs. The GNRs were then purified by ultracentrifugation. Narrow GNRs with a width of 10-30 nm were generated. CNTs have also been unzipped by catalytic cutting ${ }^{99}$ and pulseburning at high current. ${ }^{100}$

As strategies for scalable fabrication of uniform CNTs and their alignment are being developed, the synthesis of GNRs by longitudinal unzipping of CNTs is also becoming a promising approach to achieve mass production of GNRs. Further improvement of the strategy could include synthesizing GNRs of uniform $W$ with narrow $W$ distribution, of defined edges with specific chirality, and of fixed number of cylinders.

\subsection{Block-copolymer lithography}

Block copolymers can self-assemble into various nanostructures, such as spheres, cylinders, lamellae, vesicles, and many other complex or hierarchical assemblies. ${ }^{101}$ The nanostructures can have a feature size between $10 \mathrm{~nm}$ and $100 \mathrm{~nm}$, depending on the design of the blocks and the number of monomer units. Benefiting from this tailorable feature, block copolymers have been widely used in nanotechnology, e.g., as lithographic tools in the semiconductor industry. ${ }^{102-105}$ Recently, block-copolymer templates have been used to produce GNRs. ${ }^{106-109}$

Self-assembled block copolymers have been used as etch masks to convert their patterns to graphene for fabrication of GNR arrays. ${ }^{106-109}$ Some early efforts include the production of 12 nm-wide GNRs ${ }^{107}$ and sub-10 nm GNRs by controlling the number of brush layers and etching time, ${ }^{108}$ but either no obvious change was observed between the GNRs and graphene flakes or no electrical characteristics were reported. Polystyreneblock-polydimethylsiloxane (PS- $b$-PDMS) is a block copolymer with a very high Flory-Huggins interaction parameter, which ensures very small periods. Complex patterns from PS- $b$-PDMS self-assembly are also attainable. ${ }^{110}$ These properties make the block copolymer suitable for the fabrication of highly aligned sub-10 nm grating patterns. ${ }^{109,111}$

Liang et al. fabricated GNR arrays with half-pitch $<10 \mathrm{~nm}$ by using a nanofabrication approach that combines nanoimprint lithography and self-assembled templates of block copolymers. ${ }^{109}$ CVD-grown graphene monolayers that were transferred onto a $300 \mathrm{~nm}-\mathrm{SiO}_{2}$-coated $\mathrm{p}+\mathrm{Si}$ wafer were used as the matrix for patterning. 

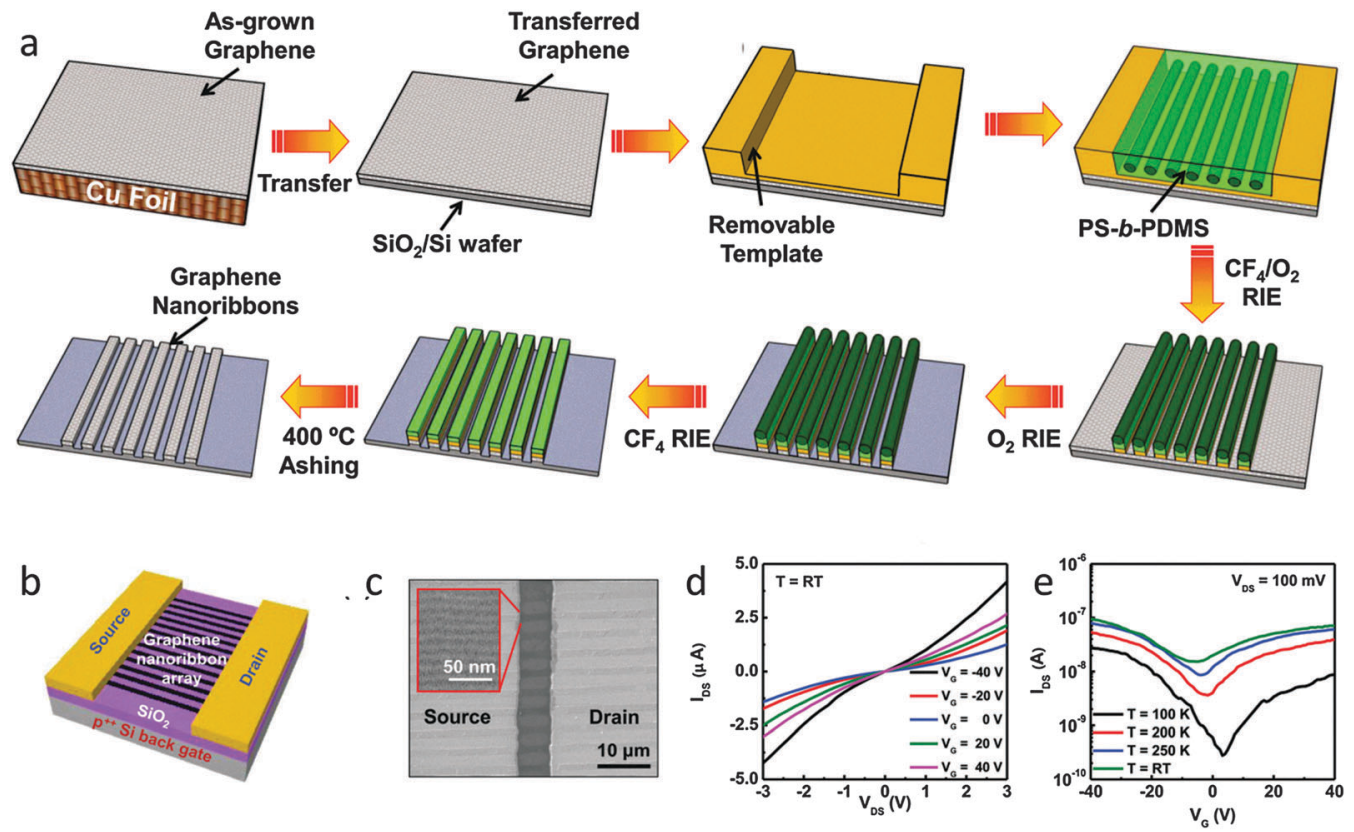

Fig. 11 (a) Schematic of processes to fabricate self-assembled PS-b-PDMS patterns and graphene nanoribbons. (b) Schematic of a multi-channel GNRFET. (c) SEM image of the channel region of the GNRFET with a magnified image of the GNR arrays. (d) Output and (e) transfer curved of the GNRFETs. (Reproduced with permission from ref. 106, Copyright 2013, Wiley-VCH Verlag GmbH \& Co.)

A poly(styrene- $b$-dimethylsiloxane) (PS- $b$-PDMS) block copolymer thin layer was spin-coated and then annealed at favorable temperature. Phase separation during annealing yielded PS and PDMS nanoscale domains (Fig. 11). Nanostructured single-layer PDMS cylinders with diameter $<10 \mathrm{~nm}$ were formed, which were surrounded by PS. ${ }^{112}$ The PS component was easily removed by a brief oxygen plasma treatment to leave PDMS cylinders to serve as etch masks that attach to the underlayer. Directional $\mathrm{CF}_{4} / \mathrm{O}_{2}$-based $\mathrm{RIE}$ was used to remove the unprotected cross-linked polymer and underlying graphene. GNRs with $W<10 \mathrm{~nm}$ were formed. Over-etching cleaned up the remaining directing structures and underlying graphene between the GNRs.

Multi-channel FETs with $\sim 50$ GNRs showed an improved $I_{\mathrm{ON}} / I_{\mathrm{OFF}}>10$, which indicates that a bandgap opened in the densely arranged graphene nanoribbons. The $I_{\mathrm{ON}} / I_{\mathrm{OFF}}$ was significantly affected by the standard deviation of the width of GNRs in the multi-channel device geometry. The block copolymer self-assembly could be controlled by altering the processing conditions, and this approach could be used to control the standard deviation of the width of GNRs.

High-density and uniform GNRs on a large area with halfpitch $<10 \mathrm{~nm}$ were produced by combining directed selfassembly of block-copolymers and nanoimprint lithography. ${ }^{109}$ The GNRs were used as active channels in multichannel fieldeffect transistors. The large variation in the width of the ribbons in the multichannel devices could cause switching behaviors to lose synchrony, and thereby result in a poor $I_{\mathrm{ON}} / I_{\mathrm{OFF}}$. GNRs with half-pitch $=8 \mathrm{~nm}$ were produced. The minimal ribbon-to-ribbon width variation was $\sim 2.4 \mathrm{~nm}$ ( $3 \sigma$ value). An $I_{\mathrm{ON}} / I_{\mathrm{OFF}}>10$ was achieved at RT; this result suggests that a bandgap opened in the GNR arrays. The $I_{\mathrm{ON}} / I_{\mathrm{OFF}}$ was among the highest values of GNRFETs with dense GNR arrays as active channels at the time.

Son et al. developed a PS- $b$-PDMS template that self-assembled into cylindrical segregating morphology, and then used the template as a lithographic tool for patterning CVD-grown graphene. ${ }^{106}$ They also used a transferred graphene sheet that had been grown using copper-catalyzed CVD. GNRs were produced with width in the range of 9-12 nm, and their field-effect and photoelectrical characteristics were also evaluated. The $I_{\mathrm{ON}} / I_{\mathrm{OFF}}$ was higher in the GNR arrays than in the graphene sheet, and was strongly dependent on temperature. GNR arrays generated 30 times more photocurrent than did a bulk graphene sheet; this difference indicates that GNRs have semiconducting properties, and further confirms that a band gap opened. Compact GNR arrays would help realize large-area graphene electronics.

The fabrication of multichannel GNRFETs using BCP lithography helps to understand the relationship between uniformity of GNRs in an array and the $I_{\mathrm{ON}} / I_{\mathrm{OFF}}$ of a multichannel GNRFET. BCP-template lithography provides a practical strategy to produce densely aligned GNR arrays with half-pitch $<10 \mathrm{~nm}$, which are desirable in future nanoelectronics.

\subsection{Narrowing of GNRs from edges}

Another strategy to produce GNR arrays is to narrow GNRs from the edges. Wang et al. ${ }^{113}$ used conventional lithographic approaches to fabricate $\sim 20-30 \mathrm{~nm}$-wide GNR arrays by electron-beam lithography, and then used gas-phase reactions to narrow them. Under optimized conditions, GNRs could be uniformly etched from edges; the authors suggested that this uniformity is due to the presence of functional groups and 
band disorders at the edge carbons, which are more chemically reactive than those within the plane. ${ }^{114}$ GNRs produced using e-beam lithography $(\sim 20 \mathrm{~nm})$ were etched from edges at a rate of $0.51 \mathrm{~nm} \mathrm{~min}{ }^{-1}\left(25 \mathrm{mTorr}_{2}\right.$ in 1 Torr $\mathrm{NH}_{3} / \mathrm{Ar}$ at $\left.800{ }^{\circ} \mathrm{C}\right)$. The etching process involves $\mathrm{O}_{2}$ oxidation of carbons into gaseous products, e.g., $\mathrm{CO}_{2}$ and $\mathrm{CO}$. The $\mathrm{C}$ atoms at edges and defect sites preferentially react with $\mathrm{O}_{2}$ molecules. ${ }^{115,116}$ The $\mathrm{NH}_{3}$ atmosphere contributed to slow down the etching rate during oxidation, ${ }^{117}$ because $\mathrm{NH}_{3}$ is efficient in reducing oxygen groups in graphene oxide, so reduction by $\mathrm{NH}_{3}$ could slow down the oxidation of graphene from edges.

Using this approach, GNR arrays with width $\leq 5 \mathrm{~nm}$ were produced. GNRFETs in which GNRs were used as active channels achieved a very high $I_{\mathrm{ON}} / I_{\mathrm{OFF}}$ of $\sim 10^{4}$ at RT; this is further evidence that a band gap opened. GNRFETs in which the active channel consisted of parallel arrays of $\sim 8 \mathrm{~nm}$-wide GNRs were also fabricated and showed an $I_{\mathrm{ON}} / I_{\mathrm{OFF}}$ of $\sim 50$ and a much higher 'on' current than did single-ribbon devices. This technique provides an important route to realizing large-scale integrated circuits based on GNRs.

\subsection{Protection at edges}

2.6.1. Graphene edge lithography based on selective ALD. Zhang's group used graphene edge lithography to allow scalable fabrication of GNR arrays and graphene nanorings. ${ }^{118}$ This approach benefits from the phenomenon that oxides grow preferentially inward from graphene edges during atomic layer deposition (ALD). The oxides that grew near the edges then serve as protective masks against dry etching. After dry etching, GNRs covered by the oxides remain, and the oxide thin layer then serves as a high- $k$ dielectric for further fabrication of GNRFETs. The authors fabricated $15 \mathrm{~nm}$-wide GNRs that had a carrier mobility of $\sim 400 \mathrm{~cm}^{2} \mathrm{~V}^{-1} \mathrm{~s}^{-1}$.

2.6.2. Meniscus-mask lithography. Tour's group proposed meniscus-mask lithography (MML), which also has potential for scalable fabrication of GNRs. ${ }^{119} \mathrm{MML}$ is a top-down lithography approach, and can fabricate GNRs with $W<10 \mathrm{~nm}$ and $\mathrm{AR}>2000$ with precise positional control from graphene sheets. First, lithography patterns were etched in the design in which GNRs produced later will form at the pattern edges. After development of the pattern, the sample was subjected to RIE to remove graphene from unprotected regions, and then a sacrificial metal layer was deposited. The resist patterns were then lifted off to leave metal patterns. A meniscus of adsorbed water remained at the foot of the metal pattern, and protected the narrow graphene region near the edge, and is the actual mask for the ultimate GNRs. The sample was again subjected to RIE to form GNRs. The metal layer was then removed by wet-etching. GNRs finally formed at the edges of the lithography patterns. During this process, a narrow region of graphene near the edges of initial patterns was protected to form narrow GNRs. The key step is the masking against RIE etching by the adsorbed water at the edges of patterns.

MML is an efficient top-down approach to produce GNRs with $W<10 \mathrm{~nm}$ and high AR; it is also scalable. By exploiting the mechanism of the technique, GNRs with an arbitrary configuration can be produced. The process does not use highresolution lithography tools. The use of Ar-RIE ensures the production of armchair edges of GNRs and consistent electronic properties in the resulting devices. Extended applications of the technique could include fabrication of nanostructures of diverse materials.

\subsection{Barrier-guided growth}

Arnold's group proposed barrier-guided chemical vapor deposition (BG-CVD) of micro- and nano-structured graphene. ${ }^{120}$ In $\mathrm{BG}-\mathrm{CVD}$, a barrier material (i.e., $10 \mathrm{~nm}$ alumina) is placed on a catalytic substrate to restrict the graphene growth to desired regions. Alumina was selected as the barrier material due to its low $\mathrm{C}$ solubility, high thermal stability and chemical inertness to both $\mathrm{C}$ and $\mathrm{Cu}^{121}$ During subsequent CVD, the selective coverage of inert oxide caused methane to decompose selectively on uncovered $\mathrm{Cu}$ surfaces, and due to the low solubility of $\mathrm{C}$ in $\mathrm{Cu},{ }^{122} \mathrm{C}$ cannot diffuse under alumina. Selective growth was ensured by the inertness of oxide and by a strong $\mathrm{Cu}-\mathrm{C}$ bonding energy of $\sim 5 \mathrm{eV} \cdot{ }^{123}$ After the processes of nucleation and lateral growth to cover the fully exposed $\mathrm{Cu}$ surface, graphene growth terminated. The patterned graphene produced by BG-CVD on the $\mathrm{Cu}$ substrate can be transferred to arbitrary substrates with the assistance of surface-coated supporting polymers.

BG-CVD is a bottom-up growth approach to laterally guided synthesis of graphene. Highly crystalline, rationally patterned micro- and nano-structured graphene were produced. In contrast to lithography approaches, high quality edges are expected.

\subsection{Nanowire lithography}

2.8.1. Inorganic-nanowire lithography. Inorganic nanowire (i-NW) lithography is a technique that uses i-NWs on graphene as physical etching masks during lithographic production of GNRs. Chemical methods synthesize i-NWs that have nearly atomically smooth edges and diameters from several tens of nanometers down to $1-2 \mathrm{~nm} .{ }^{124-129}$

Bai et al. reported the first use of i-NWs and an etch mask to produce GNRs (Fig. 12a). ${ }^{12} \mathrm{Si}$ i-NWs were grown using a nanocluster-mediated vapor-liquid-solid growth approach and then placed on top of graphene by using an AFM tip (Veeco Dimension 5000). The samples were dipped into isopropyl alcohol to introduce capillary force to ensure tight contact and then dried under blown $\mathrm{N}_{2}$. The samples were then exposed to $\mathrm{O}_{2}$ plasma to selectively remove the unprotected part of graphene and leave GNRs beneath the i-NWs, which served as protective masks against RIE etching. The initial etching was nearly anisotropic to transfer the shadow to GNRs; extended $\mathrm{O}_{2}$ plasma exposure further narrowed the width by causing lateral etching of the shadowed area that was not in direct contact with the underlying graphene. GNRs with width from $30 \mathrm{~nm}$ down to $6 \mathrm{~nm}$ were produced in this way. The protective i-NWs can be easily removed by brief sonication. This approach of using chemically synthesized inorganic nanowires as etch masks allows rational fabrication of GNRs. GNRs with width $<10 \mathrm{~nm}$ were obtained; they had a band gap of $100 \mathrm{meV}$. FETs that used the GNR as the active channel had a $I_{\mathrm{ON}} / I_{\mathrm{OFF}}$ of $\sim 150$. Similar approaches were developed subsequently. ${ }^{130}$ 
a
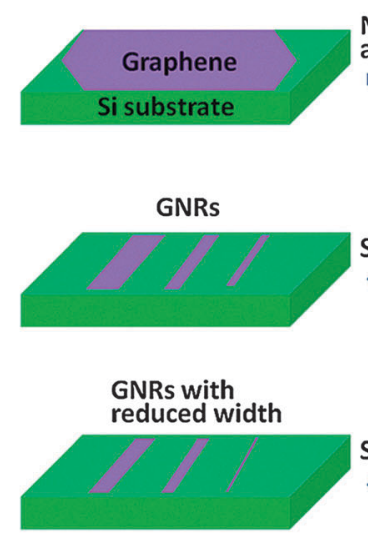
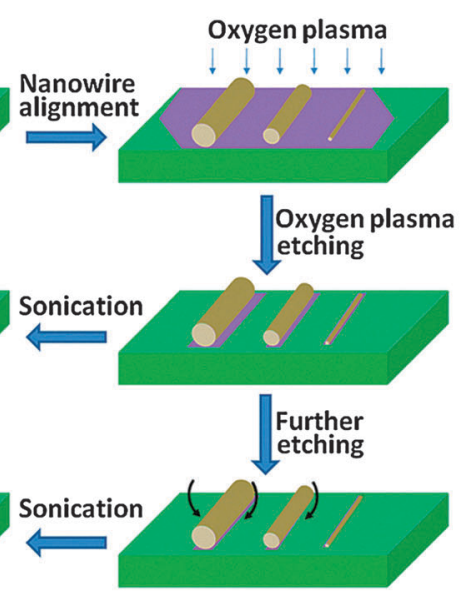

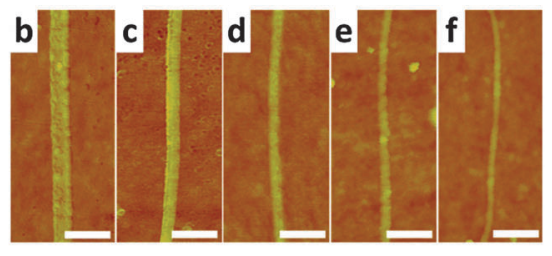

g

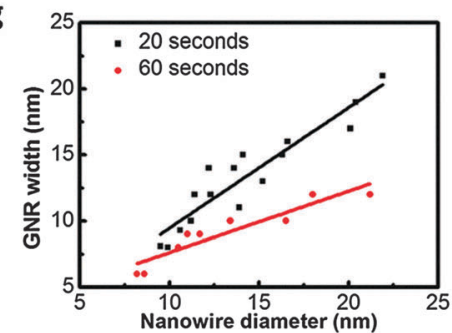

Fig. 12 i-NW lithography. (a) Schematic demonstration of the nanowire lithography process using nanowires with various diameters for the fabrication of GNRs with various diameters. (b-f) AFM images of the produced GNRs with width from $31 \mathrm{~nm}$ to $6 \mathrm{~nm}$. Scale bars: $100 \mathrm{~nm}$. (g) Correlations between GNR width and nanowire diameter for GNRs produced by oxygen plasma treatment for $20 \mathrm{~s}$ (black) and $60 \mathrm{~s}$ (red). (Reproduced with permission from ref. 12, Copyright 2009, The American Chemical Society.)

The simple use of an insulating i-NW etch mask as a gate insulator was demonstrated by Liao et al. ${ }^{131}$ They used thermally grown zirconium oxide $\left(\mathrm{ZrO}_{2}\right)$ nanocrystals with lengths of several tens of micrometers and diameters of $40-100 \mathrm{~nm}$. $\mathrm{ZrO}_{2}$ has a wide bandgap (5.1-7.8 eV) and a high dielectric constant, and is a good gate insulating material in FETs. ${ }^{132,133}$ The $\mathrm{ZrO}_{2}$ i-NWs were physically transferred to the graphene surface by contact printing and then aligned using shear force during a sliding process. Capillary force was then introduced to form close contact between $\mathrm{ZrO}_{2}$ i-NWs and graphene. A long RIE etching time of $160 \mathrm{~s}$ at $40 \mathrm{~W}$ was used to achieve severe lateral etching to leave narrow GNRs. $50 \mathrm{~nm}$-diameter i-NWs usually produce an underlying $15 \mathrm{~nm}$-wide GNR. A later-deposited electrode that was not in contact with the GNR served as a gate electrode. An RT $I_{\mathrm{ON}} / I_{\mathrm{OFF}}$ of $\sim 12$ was calculated from the transfer curves at a drain voltage of $\sim 0.1 \mathrm{~V}$ (Fig. 12b). The device can be evaluated either as a bottom-gated transistor insulated by $\mathrm{SiO}_{2}$ or as a top-gated device insulated by $\mathrm{ZrO}_{2} \mathrm{NW}$. Compared with a bottom-gated device, the use of a high- $k$ i-NW gate insulator significantly reduced the operating voltage from $10 \mathrm{~V}$ to $2 \mathrm{~V}$ (Fig. 12c). A high field-effect mobility of $\sim 1300 \mathrm{~cm}^{2} \mathrm{~V}^{-1} \mathrm{~s}^{-1}$ was achieved in the devices; this result indicates that the GNRs were of high quality and had relatively smooth edges.

The technique was further modified by using a conductive $\mathrm{i}-\mathrm{NW}$ which later served as a conductive gate. $\mathrm{An} \mathrm{HfO}_{2}$ thin layer surrounding the $\mathrm{i}-\mathrm{NW}$ was then deposited, and later used as the gate insulator. ${ }^{134}$ The wide bandgap $(\sim 5.8 \mathrm{eV})$ and high dielectric constant $(\sim 25)$ made $\mathrm{HfO}_{2}$ an excellent gate dielectric. ${ }^{135}$ ALD allows deposition of a high-quality $\mathrm{HfO}_{2}$ layer as thin as $2 \mathrm{~nm}$ to serve as a gate insulator layer between the conductive NW and the GNR active channel; this layer allowed negligible tunneling leakage current in the device during a gate voltage sweep between $-1 \mathrm{~V}$ and $+1 \mathrm{~V}$. The device had an on-current of $27 \mu \mathrm{A}$ (at $V_{\mathrm{d}}=1 \mathrm{~V}$ and $V_{\mathrm{g}}=-1 \mathrm{~V}$ ) and RT $I_{\mathrm{ON}} / I_{\mathrm{OFF}}=70$ under ambient conditions; these measurements are consistent with those of a 10 nm-wide GNR. ${ }^{33,136}$
A three-dimensional finite element method was used to evaluate the capacitance of the capacitor structure of a $30 \mathrm{~nm}$ $\mathrm{Si} / \mathrm{HfO}_{2}$ core/shell nanowire with a $0.5 \mathrm{~nm} \mathrm{SiO}{ }_{x}$ core and a $2 \mathrm{~nm}$ $\mathrm{HfO}_{2}$ shell. The electrostatic capacitance was calculated to be $3530 \mathrm{nF} \mathrm{cm}^{-2}$, which is even larger than the quantum capacitance $\left(\sim 2000 \mathrm{nF} \mathrm{cm}^{-2}\right)$ of graphene. ${ }^{137}$ The calculated hole mobility in the GNR device was $880 \mathrm{~cm}^{2} \mathrm{~V}^{-1} \mathrm{~s}^{-1}$.

Bilayer GNRs have also been fabricated using i-NW lithography. ${ }^{138}$ This work also reported that the band gap of GNRs can be further increased by breaking the inversion symmetry of a bilayer GNR. Core/shell structured Si NWs with a $2 \mathrm{~nm}$-thick $\mathrm{Al}_{2} \mathrm{O}_{3}$ layer uniformly coated on the surface were used as lithographic masks for GNRs. Due to the easy breakdown of the $\mathrm{SiO}_{2}$ layer after $\mathrm{O}_{2}$ plasma treatment, additional $60 \mathrm{~nm}$ $\mathrm{HfO}_{2}$ layers were deposited after the production of GNRs to enhance the dielectric layer. The NW lithographic bilayer GNR was thus bottom-gated with a $300 \mathrm{~nm}$-thick $\mathrm{SiO}_{2}$ layer and topgated with the $\mathrm{Si} / \mathrm{Al}_{2} \mathrm{O}_{3}$ core/shell $\mathrm{NW}$ and the $60 \mathrm{~nm} \mathrm{HfO}_{2}$ layer. As a bottom-gated device, the GNRFETs had an $I_{\mathrm{ON}} / I_{\mathrm{OFF}}$ of $\sim 10$, which is similar to previous reports. This ratio was increased to 70 by breaking the inversion symmetry.

The application of a vertical electric field is probably an efficient way to further increase the $I_{\mathrm{ON}} / I_{\mathrm{OFF}}$. The GNRFET produced using an extended etching time to narrow the GNRs showed an $I_{\mathrm{ON}} / I_{\mathrm{OFF}}$ of $\sim 400$ at RT; the ratio was increased to $\sim 3000$ by applying a vertical electric field. This ratio is 30 times higher than the previous record high for bilayer graphene. ${ }^{139}$

Breaking inversion symmetry by introducing a vertical electric field increases the band gap of bilayer GNRs, but not of singlelayer GNRs. The difference in response occurs because the two structures have distinct bandgap structures. Although bilayer graphene has a gapless band structure with conduction and valence bands in contact at a $K$ point, a finite band gap can be opened by applying a perpendicular electric field to the graphene plane; under this condition the conductive band moves upward and the valence band moves downward. ${ }^{8}$ The widening of the 
bandgap of GNRs by introducing a vertical electric field was predicted theoretically. ${ }^{140,141}$

Zhou et al. introduced the use of on-wire lithography (OWL), which is usually used to produce nanowires with nanogaps, for the production of GNRs. The approach is especially effective in producing GNRs with gaps of $<10 \mathrm{~nm} .{ }^{142}$

Compared with conventional EBL, i-NW lithography can produce GNRs with smoother edges because the resolution of the former method is limited and the latter has smooth edges of etch masks, i.e., nanowires usually have very smooth edges. Chemically synthesized i-NWs are usually used for i-NW lithography. However, this approach is only feasible for small-scale fabrication due to the difficulty in alignment; the use of this technique for mass production of GNR circuits is not currently feasible.

The inherent properties of i-NWs, such as the nanoscale width and smooth edges, qualify them for use as templates in the fabrication of GNRs. However, although this is a simple approach, the technique is usually limited by the low AR of NWs and the difficulty of aligning i-NWs after growth. Mass production of i-NWs with controllable alignment remains a difficult challenge. Even though several approaches have been proposed, they mainly showed the possibility of aligning postsynthesized NWs with a low AR, but the use of such aligned NWs as etch masks is not feasible for the production of longcontinuous, digitally positioned GNRs. Mass production of GNRs using NW lithography is thus limited by the currently available techniques for i-NWs.

2.8.2. Electrohydrodynamic nanowire lithography. Although i-NWs are perfect etch masks for the production of narrow GNRs with smooth edges, precise alignment of i-NWs on a large area remains a problem. Several approaches have been proposed for alignment of i-NWs after their growth, ${ }^{143,144}$ by utilizing electric and magnetic fields, ${ }^{145-148}$ shear forces, ${ }^{149-155}$ biorecognition ${ }^{156,157}$ and block copolymer self-assembly. ${ }^{158}$ However, i-NWs usually have a low AR, which hinders the fabrication of long continuous nanostructures with individual control over position, and alignment of each NW remains difficult.

The electrohydrodynamic nanowire printing (e-NW) printing technique is a probable solution, ${ }^{159}$ which prints long, continuous organic nanowires with digitally controlled precision of alignment. This is realized using home-built equipment that consists of two major parts (Fig. 13a). One part is the injector, composed of a syringe pump, a syringe with a metallic nozzle and a high-voltage (kilovolt-level) supplier connected to the nozzle. This part is similar to an electro-spinning system. The other part of the equipment is a horizontally placed collector, which is electrically grounded and can move horizontally under computerized control of speed and direction. During e-NW printing, the syringe is filled with polymer solution. The syringe pump controls the injection rate of polymer solution. The organic solvent is vaporized in the high electric field and solid state e-NWs finally arrive at the collector (Fig. 13a inset).

To successfully print e-NWs with digitally controlled patterns, special attention should be paid to the viscosity of the solution, injection rate of the solution, supplied voltage, tip-to-collector
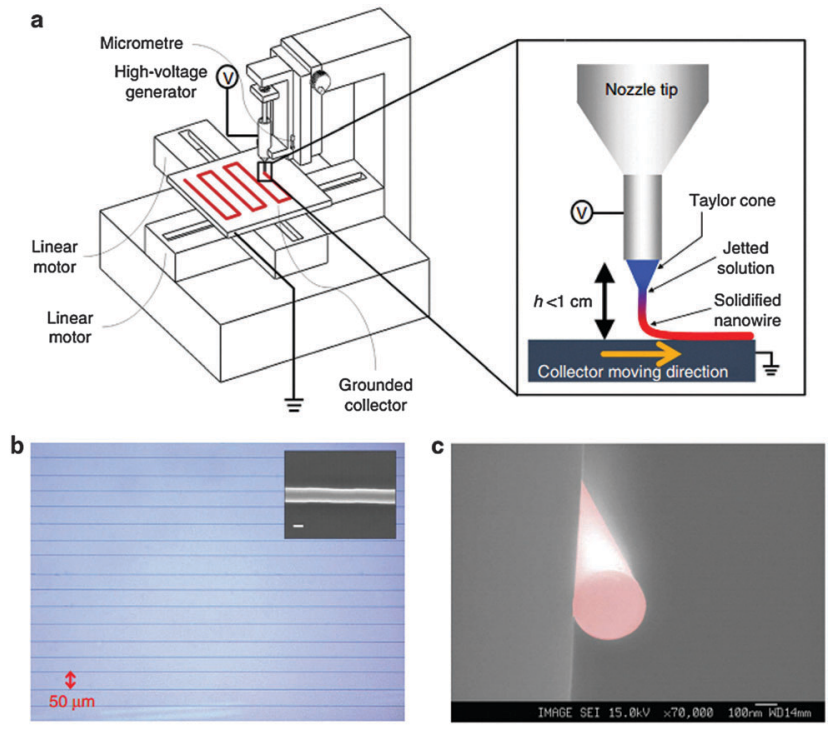

Fig. 13 (a) Schematic of an e-NW printing system. (b) Well-aligned PVK NWs with a spacing of $\sim 50 \mu \mathrm{m}$ and a diameter of $290 \mathrm{~nm}$ (inset, scale bar, $200 \mathrm{~nm}$ ). (c) SEM image of the cross-sectional view of a PVK NW. (Reproduced with permission from ref. 144, Copyright 2013, Nature Publishing Group.)

distance $D_{\mathrm{TC}}$ and the moving speed and direction of the collector. The tip-to-collector distance must be optimized (e.g., $2.5 \mathrm{~mm}$ for PVK). If $D_{\mathrm{TC}}$ is much shorter than this value, vaporization of solvents is insufficient, so the jet is still liquid when it strikes the platform. If $D_{\mathrm{TC}}$ is too long, the printed NWs form coils, as occurs in the electrospinning process. At $D_{\mathrm{TC}}=2.5 \mathrm{~mm}$, well-aligned PVK NWs are printed, with an average diameter of $290 \mathrm{~nm}$ (Fig. 13b, inset). The resultant electrodynamically produced nanowires (e-NWs) are perfectly circular in cross-section (Fig. 13c).

The well-aligned e-NWs can be used as etch masks of GNRs (Fig. 14). As a benefit of the digital control of the moving direction and speed of the collector, the e-NWs can be printed in various alignments including parallel lines with different pitch, grids, ladders, and stars (Fig. 15a-e). The diameter of e-NWs can be easily controlled by modulating the concentration of the polymer solution. The diameter of the e-NWs can be controlled from several hundreds of nanometers down to several tens of nanometers (Fig. 15f-j). Oxygen plasma removes the unprotected regions of graphene to transfer the patterns of e-NWs to the produced underlying GNRs (Fig. 15k-o). ${ }^{160-162}$

The various alignments of the e-NWs can be used as etch masks on graphene to transfer these patterns to the produced GNRs. Due to the perfectly circular cross-section of the e-NWs, the contact between the e-NW and the underlying graphene is very narrow. An extended etch time causes isotropic, lateral etching of shadowed areas that are not in direct contact with e-NW; the result is a very narrow GNR. Electrical characteristics were evaluated for the GNRs etched for various durations (Fig. 16a-c). An $I_{\mathrm{ON}} / I_{\mathrm{OFF}} \sim 70$ was achieved in a FET constructed from a GNR etched for $24 \mathrm{~s}$ (Fig. 16c). A narrow GNR with a width of $\sim 9 \mathrm{~nm}$ was achieved (Fig. 16d). The band gap of 


.

Fig. 14 Schematic of the e-nanowire lithographic process for the fabrication of GNR arrays. (Reproduced with permission from ref. 145, Copyright 2014, Wiley-VCH Verlag GmbH \& Co.)

this GNR was $100 \mathrm{meV}$ at RT. Controlled alignment of e-NWs allowed fabrication of GNRFET arrays on a wafer scale (Fig. 16e).

\section{GNRs for electronics and photonic applications}

Scalable production techniques potentially enable integration of densely aligned GNRs into circuits as logic, photonic and sensing components. Controlled alignment of GNRs on a large area further permits a variety of applications of GNRs. Numerous papers have reported the development of GNRFETs as discussed above, which will not be covered in the following discussions.

\subsection{Applications in field-effect transistors}

The most important purpose to produce GNRs is to open a bandgap and give them the potential to replace silicon-based FETs in future electronics. Intensive research in recent years has significantly improved the electrical characteristics of GNRFETs, such as their field-effect mobility, on/off current ratio and device density.

Field effect mobilities $>1000 \mathrm{~cm}^{2} \mathrm{~V}^{-1} \mathrm{~s}^{-1}$ have been achieved in high-quality GNRs with smooth edges. ${ }^{35,46,76,78,130,131}$ The on/off current ratio is increased by reducing the GNR width. At RT, e-beam lithographic approaches produce GNRs that have $I_{\mathrm{ON}} / I_{\mathrm{OFF}}$ values $>10 ;^{34,36}$ unzipping and NWL approaches could improve the $I_{\mathrm{ON}} / I_{\mathrm{OFF}}$ to $>100 .{ }^{12,95}$ Bilayer GNRs in a vertical electrical field achieved an $I_{\mathrm{ON}} / I_{\mathrm{OFF}}$ of $\sim 3000 .{ }^{138}$ So far the highest $I_{\mathrm{ON}} / I_{\mathrm{OFF}}$ values of $10^{5} \leq I_{\mathrm{ON}} / I_{\mathrm{OFF}} \leq 10^{7}$ have been reported in chemically synthesized GNRs by Dai's group..$^{21,56}$

GNRFET arrays have been massively produced on a wafer scale. ${ }^{65}$ High-density transistor arrays with 10000 top-gated graphene transistors were fabricated on a $0.24 \mathrm{~cm}^{2} \mathrm{SiC}$ chip; the density of $40000 \mathrm{~cm}^{-2}$ was a record for graphene electronic devices. ${ }^{76}$ However, these superior properties are usually reported separately; future development of the research field needs a scalable fabrication technique that can produce GNR arrays with all these superior characteristics at the same time.

\subsection{Application in logic circuits}

The crossbar architecture is a kind of nanoscale array constructed from two orthogonal sets of parallel nanoscale elements (e.g., SiNWs, CNTs, GNRs), in which every crossing point element can be regarded as an electronic device. Gholipour et al. proposed a crossbar-architecture logic circuit based entirely on Schottky-barrier GNRFETs; ${ }^{163}$ parallel GNRs formed the channel and the metal formed three terminals. Schottkybarrier type graphene nanoribbon field-effect transistors (SB-GNRFETs) were fabricated at the cross points of the GNRs and the metallic gates. The GNR-based crossbar circuits are much superior to conventional CMOS circuits in low-power applications. Habib et al. proposed a graphene nanoribbon tunneling ternary content addressable memory configuration (GNTCAM) based on graphene nanoribbon crossbar (xGNR) structures. ${ }^{164} \mathrm{~A}$ crossbar of two vertical GNRs constitutes an xGNR device that has negative differential resistance (NDR) with multiple peak and valley currents (Fig. 17a), ${ }^{165}$ which can be used as a binary latch or multi-state latch to store information in GNTCAM cell elements. In simulations GNTCAMs were up to 1.82 times denser, up to 1.6 times faster and required as little as 1/9.42 times as much stand-by power consumption than $16 \mathrm{~nm}$ CMOS TCAMs. These investigations revealed the great potential of GNRs to realize low-power high-density nanoscale circuits.

\subsection{Applications in sensors}

3.3.1. Flexible strain sensors. When strain is applied to graphene, structural deformation occurs, which changes the electrical conductivity. The electrical properties of graphene are very sensitive to this kind of mechanical deformation. Based on this principle, two-terminal graphene devices can be fabricated to serve as a strain sensor to collect electrical signal variations. Wang et al. demonstrated a GNR-based flexible strain sensor. ${ }^{166}$ They transferred patterned graphene ribbons to pre-strained PDMS, and almost perfect ripple GNRs on the elastomeric 

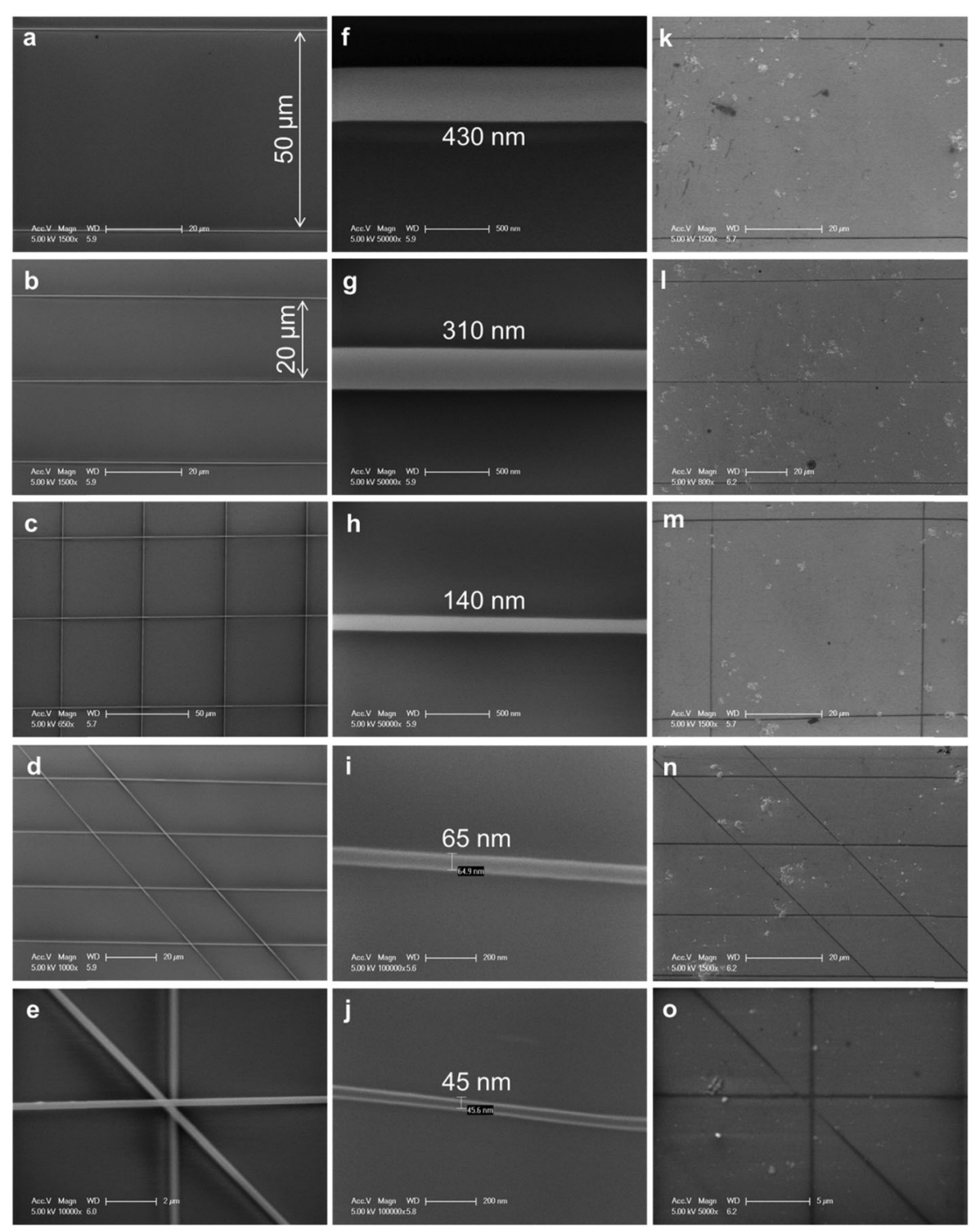

Fig. 15 SEM images of (a-e) various patterns of PVK NWs as obtained from e-NW printing, (b) PVK NWs with various diameters from several hundreds of nanometers down to several tens of nanometers, and (c) GNR patterns as etched from the PVK NW masks of (a-e). (Reproduced with permission from ref. 145, Copyright 2014, Wiley-VCH Verlag GmbH \& Co.)

PDMS substrate were then formed after releasing the stress (Fig. 17b). A Raman shift of the $2 \mathrm{D}$ peak by $\sim 15 \mathrm{~cm}^{-1}$ was observed due to the compressive strain induced after buckling. The response to external strain is linear, which guarantees the feasibility of using GNRs in future strain sensors.

3.3.2. Gas sensors. Sensing of gaseous molecules is essential in many circumstances. The large surface-to-volume ratio of graphene allows good adsorption of various gaseous molecules. The mechanism of sensing gas in graphene and graphenebased materials (e.g., GNR, GO) can be attributed to the change in the electrical properties of graphene when it is doped with adsorbed molecules. Joshi et al. reported the first fabrication of a gas sensor based on GNRs by CVD. ${ }^{167}$ They compared GNRs and graphene sheets as key components in the sensor to observe the change in electrical resistance in different gas atmospheres.
Several kinds of gas molecules were monitored, including $\mathrm{NO}_{2}, \mathrm{O}_{2}$ and $\mathrm{CO}$. Reductive gases like $\mathrm{CO}$ and oxidative gases like $\mathrm{O}_{2}$ and $\mathrm{NO}_{2}$ contribute oppositely to GNRs' resistance due to the differences in charge transfer between the sensor and gas molecules. To improve the speed and sensitivity of the GNR sensor, one method is to increase the surface sensitivity of GNRs by assembling them into a porous network structure. One group proposed a hydrogen and ammonia sensor based on Pd-functionalized multi-layer graphene nanoribbon (MLGN) networks. ${ }^{168,169}$ MLGNs are fabricated by a series of chemical processes, and then deposited on a layer of Pd with a thickness of $1 \mathrm{~nm}$ by e-beam evaporation; this layer acts as a catalyzer to improve the sensitivity to hydrogen. Using this structure, the GNRs showed $77 \%$ change in resistance relative to the initial resistance at $8000 \mathrm{ppm}_{2}$ and $70.8 \%$ at $50 \mathrm{ppm} \mathrm{NH}_{3}$. 

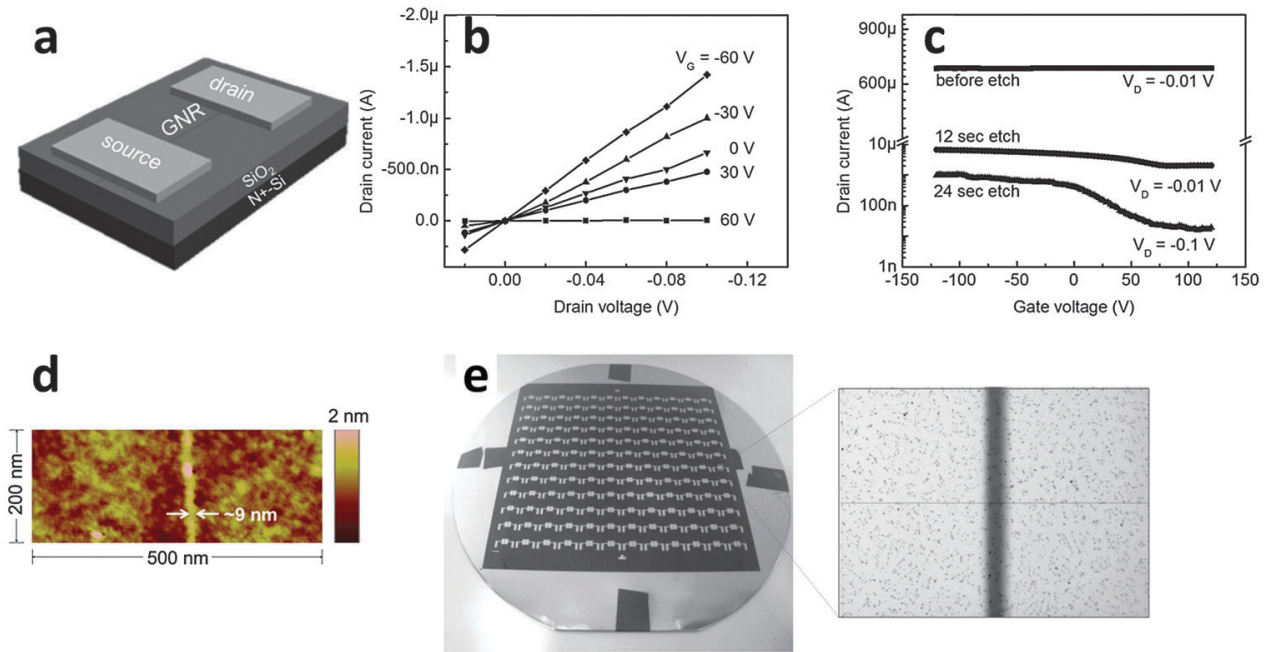

Fig. 16 (a) Architecture of a GNRFET. (b) Output characteristics and (c) transfer characteristics of a GNRFET. (d) AFM image of a 9 nm-wide GNR region. (e) Photograph of 144 GNRFETs on a 4-inch wafer with the protective PVK NW not removed. (Reproduced with permission from ref. 145, Copyright 2014, Wiley-VCH Verlag GmbH \& Co.)

a

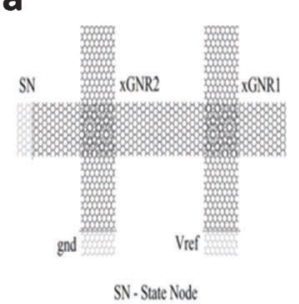

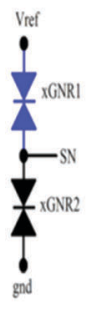

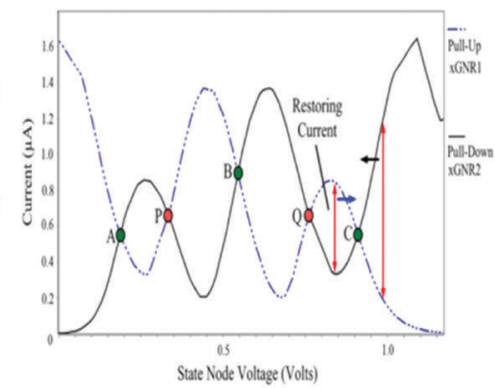

b

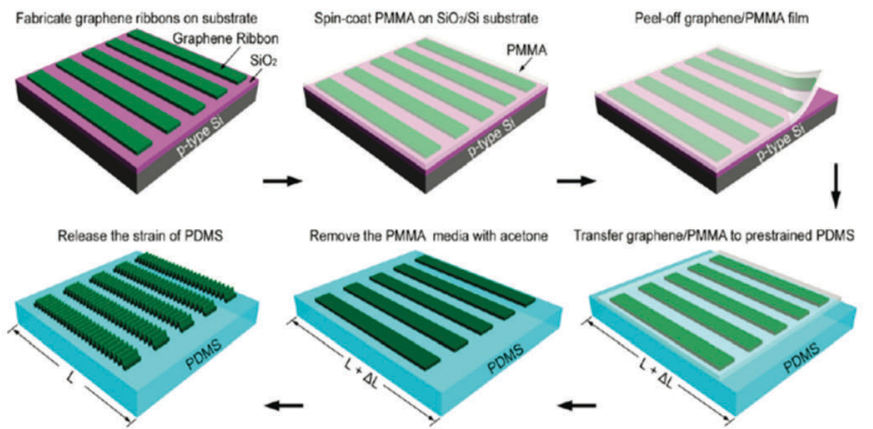

C
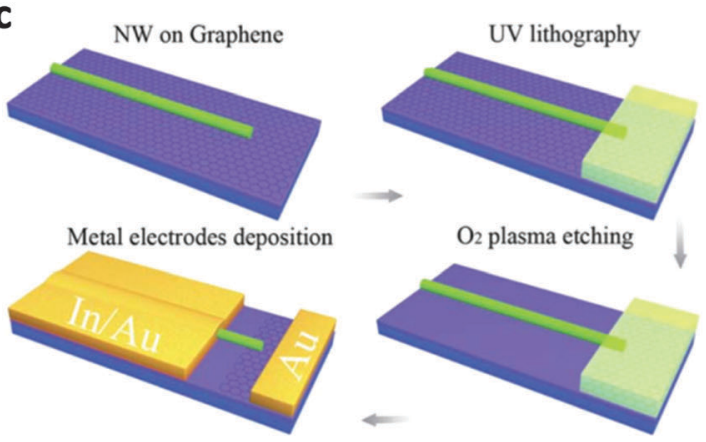

d

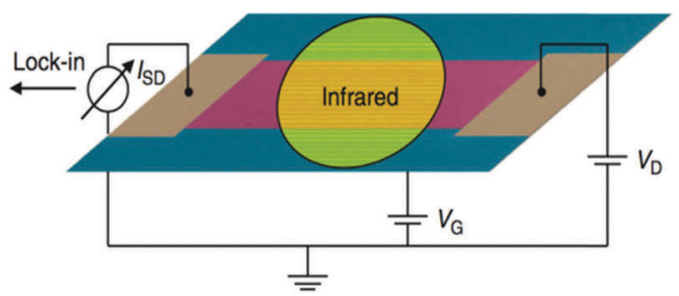

Fig. 17 Application of GNRs. (a) Application of GNRs in cross-bar shaped arrays. (Reproduced with permission from ref. 165, Copyright 2014, Elsevier.) (b) Process for fabricating ripple graphene ribbons for use in strain sensors. (Reproduced with permission from ref. 166, Copyright 2011, The American Chemical Society.) (c) Schematic of the fabrication of a GNR/SNW heterojunction LED. (Reproduced with permission from ref. 180, Copyright 2011, The Royal Society of Chemistry). (d) Schematic illustration of an IR detector based on GNRs. (Reproduced with permission from ref. 186, Copyright 2013, Nature Publishing Group.)

3.3.3. Electrochemical sensors. Several GNR-based sensors have been reported for the detection of ascorbic acid ${ }^{170}$ uric acid ${ }^{170}$ nifedipine, ${ }^{171} \mathrm{pH},{ }^{172}$ cysteine,${ }^{173}$ dobutamine, ${ }^{174}$ amino acids, ${ }^{175}$ glucose,${ }^{176}$ DNA, ${ }^{177}$ and 1-hydroxypyrene. ${ }^{178}$ In these sensors, ${ }^{170-179}$ GNRs were mainly fabricated by unzipping multiwalled carbon nanotubes and lithographic CVD graphene, except in one case ${ }^{173}$ in which they were made from graphite by Hummer's method. In most electrochemical sensors, GNRs or graphene oxide nanoribbons (GONRs) are used to decorate the electrode, which is normally composed of glassy carbon. To improve the ability to detect specific molecules, oxygenated groups must be generated on the GNR or GONR surface. Some sensors ${ }^{172,177}$ have been fabricated with a two-terminal GNRFET structure. Furthermore, a kind of DNA translocation sensor has 
been realized in the GNRFET structure with nanopores; it benefits from the relatively limited dimension of GNRs. ${ }^{177}$

\subsection{Optoelectronics applications}

3.4.1. Light-emitting diodes (LED). Ye et al. reported the fabrication of light-emitting diodes based on GNR/semiconductor NW (SNW) heterojunctions (Fig. 17c). ${ }^{180}$ A semiconductor NW was placed on a graphene sheet on a $\mathrm{SiO}_{2}$-coated highly doped $\mathrm{Si}$ substrate. A photoresist that covers one end of the SNWs was patterned by photolithography and lift off, and then treated with oxygen plasma to remove the unprotected graphene. The photoresist was then removed, and contact electrodes were deposited. After these processes, a two-terminal structured GNR/SNW heterojunction device was formed with the underlying GNRs covered by the SNWs. The face-to-face contact between GNRs and SNWs ensured a large active region. Various SNWs were tested, including $\mathrm{ZnO}, \mathrm{CdS}$ and CdSe. Due to the different bandgaps of these materials, the emitting light wavelength varied from ultraviolet $(380 \mathrm{~nm})$ to green $(513 \mathrm{~nm})$ to red $(705 \mathrm{~nm})$. In this device, recombination of electron-hole pairs mainly occurs in the SNW.

3.4.2. GNR IR photodetectors. Chitara et al. reported the first fabrication of a GNR infrared (IR) photodetector, ${ }^{181}$ after experimental investigation of the spatially resolved photocurrent of GNR devices by Stützel et al. ${ }^{182}$ Both GNRs and reduced graphene oxide (RGO) were used in the fabrication of the GNR IR photodetector. GNRs and RGO were synthesized using methods proposed by Higginbotham ${ }^{183}$ and Park, ${ }^{184}$ respectively. After absorbing light, electron-hole pairs are generated at the Schottky barrier region and then immediately pulled apart by the built-in electrical field to cause pronounced photocurrent. Responsivity $R$ and external quantum efficiency (EQE) are the important parameters of photodetectors. The GNR-based detector showed $R=1 \mathrm{~A} \mathrm{~W}^{-1}$ and $\mathrm{EQE}=80 \%$ in the mid- to near-IR spectra; the RGO photodetector achieved $R=4 \mathrm{~mA} \mathrm{~W}^{-1}$ and $\mathrm{EQE}=0.3 \%$ only. These results strongly demonstrate that GNRs are suitable candidates for use in highly responsive and highly efficient photodetectors.

Another important category of GNR IR photodetectors is based on plasmon resonance by periodic graphene nanoribbon or microribbon arrays with sub-wavelength feature sizes that improve their sensitivity. Ju et al. reported the first investigation of tunable plasmon excitation and light-plasmon coupling at terahertz frequencies in graphene microribbon arrays. ${ }^{185}$ The authors demonstrated that the plasmon resonance caused by incident light can be easily modulated by electrostatic doping (gate voltage) and the ribbon width; this modulation can control the absorption spectra. The results showed strong terahertz radiation, with $>13 \%$ absorbance of the plasmon resonance.

Freitag et al. demonstrated the first GNR-based infrared photodetector that exploits the plasmon-light coupling mechanism (Fig. 10d). ${ }^{186}$ CVD-grown single layer graphene was patterned into GNRs using EBL. GNRs with various widths ranging from $80 \mathrm{~nm}$ to $200 \mathrm{~nm}$ were evaluated for plasmon resonance. However, the photocurrent must be increased. A sophisticated device structure is required to improve the photoresponse.

\section{Conclusions and future prospects}

We have reviewed recent progress in the fabrication of GNRs, especially the general processes of methods that show promise for large-scale production of GNRs. The merits and drawbacks of each technique were also discussed. Top-down approaches, such as EBL, NWL, and BCP-template lithography, use aligned nano-patterns as protective masks to cover portions of a graphene sheet, followed by selective removal of unprotected regions to leave nanostructured graphene. Top-down processes are easy and direct ways to achieve scalable fabrication of GNRs. However, the etching process usually entails intense reactions, which produce rough edges with dangling bonds, defects, and various functional groups linked to the edges of GNRs; these rough edges degrade the electrical characteristics. In contrast, bottom-up grown GNRs from nanostructured sites can have high-quality edges and relatively better charge carrier mobility, but the patterning of nanostructured sites still requires topdown approaches, which have limited resolution, and are slow and expensive. Therefore, bottom-up approaches for scalable production of GNRs are inevitably combined with top-down approaches. More efforts are still needed to achieve addressable GNRs on a large area with individually controllable GNR width and atomically smooth edges.

\section{Acknowledgements}

This work was supported by the Center for Advanced SoftElectronics funded by the Ministry of Science, ICT and Future Planning, Korea, as Global Frontier Project (Code No. 2014M3A6A5060947).

\section{Notes and references}

1 K. S. Novoselov, A. K. Geim, S. V. Morozov, D. Jiang, Y. Zhang, S. V. Dubonos, I. V. Grigorieva and A. A. Firsov, Science, 2004, 306, 666.

2 F. Schwierz, Nature, 2011, 472, 41.

3 L. Ma, J. Wang and F. Ding, ChemPhysChem, 2013, 14, 47.

4 T.-H. Han, Y. Lee, M.-R. Choi, S.-H. Woo, S.-H. Bae, B. H. Hong, J.-H. Ahn and T.-W. Lee, Nat. Photonics, 2012, 6, 105.

5 S.-J. Byun, H. Lim, G.-Y. Shin, T.-H. Han, S. H. Oh, J.-H. Ahn, H. C. Choi and T.-W. Lee, J. Phys. Chem. Lett., 2011, 2, 493.

6 S. Bae, H. Kim, Y. Lee, X. Xu, J.-S. Park, Y. Zheng, J. Balakrishnan, T. Lei, H. R. Kim, Y. I. Song, Y.-J. Kim, K. S. Kim, B. Özyilmaz, J.-H. Ahn, B. H. Hong and S. Iijima, Nat. Nanotechnol., 2010, 5, 574.

7 Z. Yan, J. Lin, Z. Peng, Z. Sun, Y. Zhu, L. Li, C. Xiang, E. L. Samuel, C. Kittrell and J. M. Tour, ACS Nano, 2012, 6, 9110 .

8 F. Schwierz, Nat. Nanotechnol., 2010, 5, 487.

9 I. Jung, H. Y. Jang and S. Park, Appl. Phys. Lett., 2013, 103, 023105.

10 J. Yang, M. Ma, L. Li, Y. Zhang, W. Huang and X. Dong, Nanoscale, 2014, 6, 13301. 
11 T. Kato and R. Hatakeyama, Nat. Nanotechnol., 2012, 7, 651. 12 J. Bai, X. Duan and Y. Huang, Nano Lett., 2009, 9, 2083.

13 Y.-W. Son, M. L. Cohen and S. G. Louie, Phys. Rev. Lett., 2006, 97, 216803.

14 L. Yang, C.-H. Park, Y.-W. Son, M. L. Cohen and S. G. Louie, Phys. Rev. Lett., 2007, 99, 186801.

15 Y.-W. Son, M. L. Cohen and S. G. Louie, Nature, 2006, 444, 347.

16 L. Pisani, J. A. Chan, B. Montanari and N. M. Harrison, Phys. Rev. B: Condens. Matter Mater. Phys., 2007, 75, 064418.

17 J. Bai, R. Cheng, F. Xiu, L. Liao, M. Wang, A. Shailos, K. L. Wang, Y. Huang and X. Duan, Nat. Nanotechnol., 2010, 5, 655.

18 M. Y. Han, B. Özyilmaz, Y. Zhang and P. Kim, Phys. Rev. Lett., 2007, 98, 206805.

19 M. Evaldsson, I. V. Zozoulenko, H. Xu and T. Heinzel, Phys. Rev. B: Condens. Matter Mater. Phys., 2008, 78, 161407.

20 A. Cresti and S. Roche, Phys. Rev. B: Condens. Matter Mater. Phys., 2009, 79, 233404.

21 X. Li, X. Wang, L. Zhang, S. Lee and H. Dai, Science, 2008, 319, 1229.

22 E. R. Mucciolo, A. H. Castro Neto and C. H. Lewenkopf, Phys. Rev. B: Condens. Matter Mater. Phys., 2009, 79, 075407.

23 D. Querlioz, Y. Apertet, A. Valentin, K. Huet, A. Bournel, S. Galdin-Retailleau and P. Dollfus, Appl. Phys. Lett., 2008, 92, 042108.

24 Y. Zhang, Y.-W. Tan, H. L. Stormer and P. Kim, Nature, 2005, 438, 201.

25 K. Wakabayashi, M. Fujita, H. Ajiki and M. Sigrist, Phys. Rev. B: Condens. Matter Mater. Phys., 1999, 59, 8271.

26 Y. Miyamoto, K. Nakada and M. Fujita, Phys. Rev. B: Condens. Matter Mater. Phys., 1999, 59, 9858.

27 M. Ezawa, Phys. Rev. B: Condens. Matter Mater. Phys., 2006, 73, 045432.

28 N. M. R. Peres, A. H. Castro Neto and F. Guinea, Phys. Rev. B: Condens. Matter Mater. Phys., 2006, 73, 195411.

29 L. Brey and H. A. Fertig, Phys. Rev. B: Condens. Matter Mater. Phys., 2006, 73, 235411.

30 Y. Ouyang, Y. Yoon, J. K. Fodor and J. Guo, Appl. Phys. Lett., 2006, 89, 203107.

31 V. Barone, O. Hod and G. E. Scuseria, Nano Lett., 2006, 6, 2748 .

32 D. A. Areshkin, D. Gunlycke and C. T. White, Nano Lett., 2007, 7, 204.

33 Z. Chen, Y.-M. Lin, M. J. Rooks and P. Avouris, Physica E, 2007, 40, 228.

34 W. S. Hwang, K. Tahy, X. Li, H. Xing, A. C. Seabaugh, C. Y. Sung and D. Jena, Appl. Phys. Lett., 2012, 100, 203107.

35 W. S. Hwang, P. Zhao, K. Tahy, L. O. Nyakiti, V. D. Wheeler, R. L. Myers-Ward, C. R. Eddy, D. K. Gaskill, J. A. Robinson, W. Haensch, H. Xing, A. Seabaugh and D. Jena, APL Mater., 2015, 3, 011101.

36 J. Sun, T. Iwasaki, M. Muruganathan and H. Mizuta, Appl. Phys. Lett., 2015, 106, 033509.

37 V. Sidorkin, E. van Veldhoven, E. van der Drift, P. Alkemade, H. Salemink and D. Maas, J. Vac. Sci. Technol., B: Microelectron. Nanometer Struct.-Process., Meas., Phenom., 2009, 27, L18.
38 D. Winston, B. M. Cord, B. Ming, D. C. Bell, W. F. DiNatale, L. A. Stern, A. E. Vladar, M. T. Postek, M. K. Mondol, J. K. W. Yang and K. K. Berggren, J. Vac. Sci. Technol., B: Microelectron. Nanometer Struct.-Process., Meas., Phenom., 2009, 27, 2702.

39 W.-D. Li, W. Wu and R. S. Williams, J. Vac. Sci. Technol., B: Nanotechnol. Microelectron.: Mater., Process., Meas., Phenom., 2012, 30, 06F304.

40 M. C. Lemme, D. C. Bell, J. R. Williams, L. A. Stern, B. W. H. Baugher, P. Jarillo-Herrero and C. M. Marcus, ACS Nano, 2009, 3, 2674.

41 D. C. Bell, M. C. Lemme, L. A. Stern, J. R. Williams and C. M. Marcus, Precision Cutting and Patterning of Graphene with Helium Ions, Nanotechnology, 2009, 20, 455301.

42 A. N. Abbas, G. Liu, B. Liu, L. Zhang, H. Liu, D. Ohlberg, W. Wu and C. Zhou, ACS Nano, 2014, 8, 1538.

43 Y. Yinxiao and R. Murali, Impact of Size Effect on Graphene Nanoribbon Transport, IEEE Electron Device Lett., 2010, 31, 237.

44 H. Raza and E. C. Kan, Phys. Rev. B: Condens. Matter Mater. Phys., 2008, 77, 245434.

45 L. M. Demers, D. S. Ginger, S. J. Park, Z. Li, S. W. Chung and C. A. Mirkin, Science, 2002, 296, 1836.

46 Y.-S. Shin, J. Y. Son, M.-H. Jo, Y.-H. Shin and H. M. Jang, J. Am. Chem. Soc., 2011, 133, 5623.

47 L. Tapaszto, G. Dobrik, P. Lambin and L. P. Biro, Nat. Nanotechnol., 2008, 3, 397.

48 K. V. Emtsev, A. Bostwick, K. Horn, J. Jobst, G. L. Kellogg, L. Ley, J. L. McChesney, T. Ohta, S. A. Reshanov, J. Rohrl, E. Rotenberg, A. K. Schmid, D. Waldmann, H. B. Weber and T. Seyller, Nat. Mater., 2009, 8, 203.

49 W. A. de Heer, C. Berger, X. Wu, P. N. First, E. H. Conrad, X. Li, T. Li, M. Sprinkle, J. Hass, M. L. Sadowski, M. Potemski and G. Martinez, Solid State Commun., 2007, 143, 92.

50 X. Li, W. Cai, J. An, S. Kim, J. Nah, D. Yang, R. Piner, A. Velamakanni, I. Jung, E. Tutuc, S. K. Banerjee, L. Colombo and R. S. Ruoff, Science, 2009, 324, 1312.

51 J. Cai, P. Ruffieux, R. Jaafar, M. Bieri, T. Braun, S. Blankenburg, M. Muoth, A. P. Seitsonen, M. Saleh, X. Feng, K. Müllen and R. Fasel, Nature, 2010, 466, 470.

52 S. Linden, D. Zhong, A. Timmer, N. Aghdassi, J. H. Franke, H. Zhang, X. Feng, K. Müllen, H. Fuchs, L. Chi and H. Zacharias, Phys. Rev. Lett., 2012, 108, 216801.

53 H. Sakaguchi, Y. Kawagoe, Y. Hirano, T. Iruka, M. Yano and T. Nakae, Adv. Mater., 2014, 26, 4134.

54 P. Ruffieux, J. Cai, N. C. Plumb, L. Patthey, D. Prezzi, A. Ferretti, E. Molinari, X. Feng, K. Müllen, C. A. Pignedoli and R. Fasel, ACS Nano, 2012, 6, 6930.

55 K. A. Simonov, N. A. Vinogradov, A. S. Vinogradov, A. V. Generalov, E. M. Zagrebina, N. Martensson, A. A. Cafolla, T. Carpy, J. P. Cunniffe and A. B. Preobrajenski, J. Phys. Chem. C, 2014, 118, 12532.

56 X. Wang, Y. Ouyang, X. Li, H. Wang, J. Guo and H. Dai, Phys. Rev. Lett., 2008, 100, 206803.

57 Y.-C. Chen, D. G. de Oteyza, Z. Pedramrazi, C. Chen, F. R. Fischer and M. F. Crommie, ACS Nano, 2013, 7, 6123. 
58 T. H. Vo, M. Shekhirev, D. A. Kunkel, M. D. Morton, E. Berglund, L. Kong, P. M. Wilson, P. A. Dowben, A. Enders and A. Sinitski, Nat. Commun., 2014, 5, 3189.

59 P. B. Bennett, Z. Pedramrazi, A. Madani, Y.-C. Chen, D. G. de Oteyza, C. Chen, F. R. Fischer, M. F. Crommie and J. Bokor, Appl. Phys. Lett., 2013, 103, 253114.

60 A. Narita, I. A. Verzhbitskiy, W. Frederickx, K. S. Mali, S. A. Jensen, M. R. Hansen, M. Bonn, S. D. Feyter, C. Casiraghi, X. Feng and K. Müllen, ACS Nano, 2014, 8, 11622.

61 O. Endo, M. Nakamura, K. Amemiya and H. Ozaki, Surf. Sci., 2015, 635, 44.

62 A. Narita, X. Feng, Y. Hernandez, S. A. Jensen, M. Bonn, H. Yang, I. A. Verzhbitskiy, C. Casiraghi, M. R. Hansen, A. H. R. Koch, G. Fytas, O. Ivasenko, B. Li, K. S. Mali, T. Balandina, S. Mahesh, S. D. Feyter and K. Müllen, Nat. Chem., 2014, 6, 126.

63 Y.-Z. Tan, B. Yang, K. Parvez, A. Narita, S. Osella, D. Beljonne, X. Feng and K. Müllen, Nat. Commun., 2013, 4, 2646.

64 A. N. Abbas, G. Liu, A. Narita, M. Orosco, X. Feng, K. Müllen and C. Zhou, J. Am. Chem. Soc., 2014, 136, 7555.

65 P. Han, K. Akagi, F. F. Canova, H. Mutoh, S. Shiraki, K. Iwaya, P. S. Weiss, N. Asao and T. Hitosugi, ACS Nano, 2014, 8, 9181.

66 H. Zhang, H. Lin, K. Sun, L. Chen, Y. Zagranyarski, N. Aghdassi, S. Duhm, Q. Li, D. Zhong, Y. Li, K. Müllen, H. Fuchs and L. Chi, J. Am. Chem. Soc., 2015, 137, 4022.

$67 \mathrm{~J}$. Wu, L. Gherghel, M. D. Watson, J. Li, Z. Wang, C. D. Simpson, U. Kolb and K. Müllen, Macromolecules, 2003, 36, 7082.

68 J. Liu, B.-W. Li, Y.-Z. Tan, A. Giannakopoulos, C. SanchezSanchez, D. Beljonne, P. Ruffieux, R. Fasel, X. Feng and K. Müllen, J. Am. Chem. Soc., 2015, 137, 6097.

69 (a) S. Kawai, S. Saito, S. Osumi, S. Yamaguchi, A. S. Foster, P. Spijker and E. Meyer, Nat. Commun., 2015, 6, 8098; (b) C. Bronner, S. Stremlau, M. Gille, F. Brauße, A. Haase, S. Hecht and P. Tegeder, Angew. Chem., Int. Ed., 2013, 52, 4422.

70 W. J. Yu, S. H. Chae, D. Perello, S. Y. Lee, G. H. Han, M. Yun and Y. H. Lee, ACS Nano, 2010, 4, 5480.

71 C. Berger, Z. Song, T. Li, X. Li, A. Y. Ogbazghi, R. Feng, Z. Dai, A. N. Marchenkov, E. H. Conrad, P. N. First and W. A. de Heer, J. Phys. Chem. B, 2004, 108, 19912.

72 C. Berger, Z. Song, X. Li, X. Wu, N. Brown, C. Naud, D. Mayou, T. Li, J. Hass, A. N. Marchenkov, E. H. Conrad, P. N. First and W. A. de Heer, Science, 2006, 312, 1191.

73 J. Hass, W. A. de Heer and E. H. Conrad, J. Phys.: Condens. Matter, 2008, 20, 323202.

74 C. Virojanadaraa, R. Yakimovaa, J. R. Osieckia, M. Syväjärvia, R. I. G. Uhrberga, L. I. Johanssona and A. A. Zakharovb, Surf. Sci., 2009, 603, L87.

75 J. Robinson, X. Weng, K. Trumbull, R. Cavalero, M. Wetherington, E. Frantz, M. LaBella, Z. Hughes, M. Fanton and D. Snyder, ACS Nano, 2010, 4, 153.

76 M. Sprinkle, M. Ruan, Y. Hu, J. Hankinson, M. Rubio-Roy, B. Zhang, X. Wu, C. Berger and W. A. de Heer, Nat. Nanotechnol., 2010, 5, 727.
77 M. Y. Han, J. C. Brant and P. Kim, Phys. Rev. Lett., 2010, 104, 056801.

78 I. Martin-Fernandez, D. Wang and Y. Zhang, Nano Lett., 2012, 12, 6175.

79 G. Wang, M. Zhang, Y. Zhu, G. Ding, D. Jiang, Q. Guo, S. Liu, X. Xie, P. K. Chu, Z. Di and X. Wang, Sci. Rep., 2013, 3, 2465.

80 J.-H. Lee, E. K. Lee, W.-J. Joo, Y. Jang, B.-S. Kim, J. Y. Lim, S.-H. Choi, S. J. Ahn, J. R. Ahn, M.-H. Park, C.-W. Yang, B. L. Choi, S.-W. Hwang and D. Whang, Science, 2014, 344, 286.

81 R. M. Jacobberger, B. Kiraly, M. Fortin-Deschenes, P. L. Levesque, K. M. McElhinny, G. J. Brady, R. R. Delgado, S. S. Roy, A. Mannix, M. G. Lagally, P. G. Evans, P. Desjardins, R. Martel, M. C. Hersam, N. P. Guisinger and M. S. Arnold, Nat. Commun., 2015, 6, 8006.

82 X. Y. Zhang, H. Li and F. Ding, Adv. Mater., 2014, 26, 5488. 83 Y. T. Lu and H. Metiu, Surf. Sci., 1991, 245, 150.

84 R. J. Hamers, U. K. Köhler and J. E. Demuth, Ultramicroscopy, 1989, 31, 10.

85 C. Pearson, M. Krueger and E. Ganz, Phys. Rev. Lett., 1996, 76, 2306.

86 J. E. Ayers and S. K. Ghandhi, J. Cryst. Growth, 1988, 89, 371.

87 H. Ago, I. Tanaka, Y. Ogawa, R. M. Yunus, M. Tsuji and H. Hibino, ACS Nano, 2013, 7, 10825.

88 A. N. Sokolov, F. L. Yap, N. Liu, K. Kim, L. Ci, O. B. Johnson, H. Wang, M. Vosgueritchian, A. L. Koh, J. Chen, J. Park and Z. Bao, Nat. Commun., 2013, 4, 2402.

89 J. Richter, Metallization of DNA, Physica E, 2003, 16, 157.

90 N. Liu, K. Kim, P.-C. Hsu, A. N. Sokolov, F. L. Yap, H. Yuan, Y. Xie, H. Yan, Y. Cui, H. Y. Hwang and Z. Bao, J. Am. Chem. Soc., 2014, 136, 17284.

91 D. V. Kosynkin, A. L. Higginbotham, A. Sinitskii, J. R. Lomeda, A. Dimiev, B. K. Price and J. M. Tour, Nat. Nanotechnol., 2008, 3, 397.

92 F. Cataldo, G. Compagnini, G. Patane, O. Ursini, G. Angelini, P. R. Ribic, G. Margaritondo, A. Cricenti, G. Palleschi and F. Valentini, Carbon, 2010, 48, 2596.

93 D. V. Kosynkin, W. Lu, A. Sinitskii, G. Pera, Z. Sun and J. M. Tour, ACS Nano, 2011, 5, 968.

94 Y. Gong, M. Long, G. Liu, S. Gao, C. Zhu, X. Wei, X. Geng, M. Sun, C. Yang, L. Lu and L. Liu, Phys. Rev. B: Condens. Matter Mater. Phys., 2013, 87, 165404.

95 L. Jiao, L. Zhang, X. Wang, G. Diankov and H. Dai, Nature, 2009, 458, 877.

96 L. Jiao, X. Wang, G. Diankov, H. Wang and H. Dai, Nat. Nanotechnol., 2010, 5, 321.

97 Z. F. Zhong, H. L. Shen, R. X. Cao, L. Sun, K. P. Li, X. R. Wang and H. F. Ding, J. Appl. Phys., 2013, 113, 174307.

98 L. Jiao, L. Zhang, L. Ding, J. Liu, H. Dai, L. Jiao, L. Zhang, L. Ding, J. Liu and H. Dai, Nano Res., 2010, 3, 387.

99 A. L. Elias, A. R. Botello-Méndez, D. Meneses-Rodríguez, V. Jehová González, D. Ramírez-González, L. Ci, E. MuñozSandoval, P. M. Ajayan, H. Terrones and M. Terrones, Nano Lett., 2010, 10, 366. 
100 W. S. Kim, S. Y. Moon, S. Y. Bang, B. G. Choi, H. Ham, T. Sekino and K. B. Shim, Appl. Phys. Lett., 2009, 95, 083103. 101 Y. Maia and A. Eisenberg, Chem. Soc. Rev., 2012, 41, 5969. 102 International Technology Roadmap for Semiconductors, ITRS 2011 Edition (http://www.itrs.net/reports.html), 2011.

103 F. S. Bates and G. H. Fredrickson, Annu. Rev. Phys. Chem., 1990, 41, 525.

104 M. Park, C. Harrison, P. M. Chaikin, R. A. Register and D. H. Adamson, Science, 1997, 276, 1401.

105 J. Y. Cheng, C. A. Ross, H. I. Smith and E. L. Thomas, Adv. Mater., 2006, 18, 2505.

106 J. G. Son, M. Son, K.-J. Moon, B. H. Lee, J.-M. Myoung, M. S. Strano, M.-H. Ham and C. A. Ross, Adv. Mater., 2013, 25, 4723.

107 L. Jiao, L. Xie and H. Dai, Nano Res., 2012, 5, 292.

108 G. Liu, Y. Wu, Y.-M. Lin, D. B. Farmer, J. A. Ott, J. Bruley, A. Grill, P. Avouris, D. Pfeiffer, A. A. Balandin and C. Dimitrakopoulos, ACS Nano, 2012, 6, 6786.

109 X. Liang and S. Wi, ACS Nano, 2012, 6, 9700.

110 J. K. W. Yang, Y. S. Jung, J.-B. Chang, R. A. Mickiewicz, A. Alexander-Katz, C. A. Ross and K. K. Berggren, Nat. Nanotechnol., 2010, 5, 256.

111 J. G. Son, A. F. Hannon, K. W. Gotrik, A. Alexander-Katz and C. A. Ross, Adv. Mater., 2011, 23, 634.

112 Y. S. Jung, J. H. Lee, J. Y. Lee and C. A. Ross, Nano Lett., 2010, 10, 3722.

113 X. Wang and H. Dai, Nat. Chem., 2010, 2, 661.

114 X. Wang, S. M. Tabakman and H. Dai, J. Am. Chem. Soc., 2008, 130, 8152.

115 S. M. Lee, Y. H. Lee, Y. G. Hwang, J. R. Hahn and H. Kang, Phys. Rev. Lett., 1999, 82, 217.

116 J. R. Hahn, H. Kang, S. M. Lee and Y. H. Lee, J. Phys. Chem. $B$, 1999, 103, 9944.

117 X. Li, H. Wang, J. T. Robinson, H. Sanchez, G. Diankov and H. Dai, J. Am. Chem. Soc., 2009, 131, 15939.

118 G. Xie, Z. Shi, R. Yang, D. Liu, W. Yang, M. Cheng, D. Wang, D. Shi and G. Zhang, Nano Lett., 2012, 12, 4642.

119 V. Abramova, A. S. Slesarev and J. M. Tour, ACS Nano, 2013, 7, 6894.

120 N. S. Safron, M. G. Kim, P. Gopalan and M. S. Arnold, Adv. Mater., 2012, 24, 1041.

121 L. M. Foster, G. Long and M. S. Hunter, J. Am. Ceram. Soc., 1956, 39, 1.

122 X. S. Li, W. W. Cai, L. Colombo and R. S. Ruoff, Nano Lett., 2009, 9, 4268.

123 H. Chen, W. Zhu and Z. Zhang, Phys. Rev. Lett., 2010, 104, 186101.

124 X. Duan and C. M. Lieber, Adv. Mater., 2000, 12, 298.

125 J. D. Holmes, K. P. Johnston, R. C. Doty and B. A. Korgel, Science, 2000, 287, 1471.

126 Y. Huang and C. M. Lieber, Pure Appl. Chem., 2004, 76, 2051.

127 Z. Huo, C.-k. Tsung, W. Huang, X. Zhang and P. Yang, Nano Lett., 2008, 8, 2041.

128 X. Lu, M. S. Yavuz, H.-Y. Tuan, B. A. Korge and Y. Xia, J. Am. Chem. Soc., 2008, 130, 8900.
129 C. Wang, Y. J. Hu, C. M. Lieber and S. H. Sun, J. Am. Chem. Soc., 2008, 130, 8902.

130 C. G. Kang, J. W. Kang, S. K. Lee, S. Y. Lee, C. H. Cho, H. J. Hwang, Y. G. Lee, J. Heo, H.-J. Chung, H. Yang, S. Seo, S.-J. Park, K. Y. Ko, J. Ahn and B. H. Lee, Nanotechnology, 2011, 22, 295201.

131 L. Liao, J. Bai, Y.-C. Lin, Y. Qu, Y. Huang and X. Duan, Adv. Mater., 2010, 22, 1941.

132 C. M. Perkins, B. B. Triplett, P. C. McIntyre, K. C. Saraswat, S. Haukka and M. Tuominen, Appl. Phys. Lett., 2001, 78, 2357.

133 Y. C. Yeo, T. J. King and C. M. Hu, J. Appl. Phys., 2002, 92, 7266.

134 L. Liao, J. Bai, R. Cheng, Y.-C. Lin, S. Jiang, Y. Huang and X. Duan, Nano Lett., 2010, 10, 1917.

135 M. Cho, J. Park, H. B. Park, C. S. Hwang, J. Jeong and K. S. Hyun, Appl. Phys. Lett., 2002, 81, 334.

136 J. W. Bai, X. Zhong, S. Jiang, Y. Huang and X. F. Duan, Nat. Nanotechnol., 2010, 5, 190.

137 I. Meric, M. Y. Han, A. F. Young, B. Özyilmaz, P. Kim and K. L. Shepard, Nat. Nanotechnol., 2008, 3, 654.

138 W. J. Yu and X. Duan, Sci. Rep., 2013, 3, 1248.

139 F. Xia, D. B. Farmer, Y. Lin and P. Avouris, Nano Lett., 2010, $10,715$.

140 B. Sahu, H. Min, A. H. MacDonald and S. K. Banerjee, Phys. Rev. B: Condens. Matter Mater. Phys., 2008, 78, 045404.

141 M. P. Lima, A. Fazzio and A. J. R. Silva, Phys. Rev. B: Condens. Matter Mater. Phys., 2009, 79, 153401.

142 X. Zhou, C. M. Shade, A. L. Schmucker, K. A. Brown, S. He, F. Boey, J. Ma, H. Zhang and C. A. Mirkin, Nano Lett., 2012, 12,4734 .

143 N. P. Dasgupta, J. Sun, C. Liu, S. Brittman, S. C. Andrews, J. Lim, H. Gao, R. Yan and P. Yang, Adv. Mater., 2014, 26, 2137.

144 M. C. P. Wang and B. D. Gates, Mater. Today, 2009, 12, 34. 145 J. Yao, H. Yan and C. M. Lieber, Nat. Mater., 2013, 8, 329.

146 P. A. Smith, C. D. Nordquist, T. N. Jackson, T. S. Mayer, B. R. Martin, J. Mbindyo and T. E. Mallouk, Appl. Phys. Lett., 2000, 77, 1399.

147 X. Duan, Y. Huang, Y. Cui, J. Wang and C. M. Lieber, Nature, 2001, 409, 66.

148 C. M. Hangarter and N. V. Myung, Chem. Mater., 2005, 17, 1320.

149 Y. Huang, X. Duan, Q. Wei and C. M. Lieber, Science, 2001, 291, 630.

150 X. Duan, C. Niu, V. Sahi, J. Chen, J. Wallace Parce, S. Empedocles and J. L. Goldman, Nature, 2003, 425, 274.

151 G. Yu, A. Cao and C. M. Lieber, Nat. Nanotechnol., 2007, 2,372 .

152 A. Tao, F. Kim, C. Hess, J. Goldberger, R. He, Y. Sun, Y. Xia and P. Yang, Nano Lett., 2003, 3, 1229.

153 Z. Fan, J. C. Ho, T. Takahashi, R. Yerushalmi, K. Takei, A. C. Ford, Y.-L. Chueh and A. Javey, Adv. Mater., 2009, 21, 3730 .

154 K. Takei, T. Takahashi, J. C. Ho, H. Ko, A. G. Gillies, P. W. Leu, R. S. Fearing and A. Javey, Nat. Mater., 2010, 9, 821. 
155 Q. Cao, H.-S. Kim, N. Pimparkar, J. P. Kulkarni, C. Wang, M. Shim, K. Roy, M. A. Alam and J. A. Rogers, Nature, 2008, 454, 495.

156 J. K. N. Mbindyo, B. D. Reiss, B. R. Martin, C. D. Keating, M. J. Natan and T. E. Mallouk, Adv. Mater., 2001, 13, 249.

157 M. Chen, L. Guo, R. Ravi and P. C. Searson, J. Phys. Chem. B, 2006, 110, 211.

158 J. Chai, D. Wang, X. Fan and J. M. Buriak, Nat. Nanotechnol., 2007, 2, 500.

159 S.-Y. Min, T.-S. Kim, B. J. Kim, H. Cho, Y.-Y. Noh, H. Yang, J. H. Cho and T.-W. Lee, Nat. Commun., 2013, 4, 1773.

160 W. Xu, H.-K. Seo, S.-Y. Min, H. Cho, T.-S. Lim, C.-y. Oh, Y. Lee and T.-W. Lee, Adv. Mater., 2014, 26, 3459.

161 W. Xu, T.-S. Lim, H.-K. Seo, S.-Y. Min, H. Cho, M.-H. Park, Y.-H. Kim and T.-W. Lee, Small, 2014, 10, 1999.

162 W. Xu, L. Wang, Y. Liu, S. Thomas, H.-K. Seo, K.-I. Kim, K. S. Kim and T.-W. Lee, Adv. Mater., 2015, 27, 1619.

163 M. Gholipour and N. Masoumi, Microelectron. J., 2014, 45, 1533.

164 K. M. M. Habib and R. K. Lake, Phys. Rev. B: Condens. Matter Mater. Phys., 2012, 86, 045418.

165 S. Khasanvis, M. Rahman and C. A. Moritz, J. Parallel Distrib. Comput., 2014, 74, 2497.

166 Y. Wang, R. Yang, Z. W. Shi, L. C. Zhang, D. X. Shi, E. Wang and G. Y. Zhang, ACS Nano, 2011, 5, 3645.

167 R. K. Joshi, H. Gomez, F. Alvi and A. Kumar, J. Phys. Chem. C, 2010, 114, 6610.

168 J. L. Johnson, A. Behnam, S. J. Pearton and A. Ural, Adv. Mater., 2010, 22, 4877.

169 J. L. Johnson, A. Behnam, Y. An, S. J. Pearton and A. Ural, J. Appl. Phys., 2011, 109, 124301.

170 A. Martína, J. H. Ferrerb, M. T. Martínezb and A. Escarpaa, Electrochim. Acta, 2015, 172, 2.

171 L. Shang, F. Q. Zhao and B. Z. Zeng, Electrochim. Acta, 2015, 168, 330 .
172 X. B. Tan, H. J. Chuang, M. W. Lin, Z. X. Zhou and M. M. Cheng, J. Phys. Chem. C, 2013, 117, 27155.

173 S. Wu, X. Q. Lan, F. F. Huang, Z. Z. Luo, H. X. Ju, C. G. Meng and C. Y. Duan, Biosens. Bioelectron., 2012, 32, 293.

174 E. Asadian, S. Shahrokhian, A. I. Zad and E. Jokar, Sens. Actuators, B, 2014, 196, 582.

175 A. Martín, P. Batalla, J. Hernández-Ferrer, M. T. Martínez and A. Escarpa, Biosens. Bioelectron., 2015, 68, 163.

176 N. S. Ismail, Q. H. Le, H. Yoshikawa, M. Saito and E. Tamiya, Electrochim. Acta, 2014, 146, 98.

177 F. Traversi, C. Raillon, S. M. Benameur, K. Liu, S. Khlybov, M. Tosun, D. Krasnozhon, A. Kis and A. Radenovic, Nat. Nanotechnol., 2013, 8, 939.

178 X. F. Shen, Y. Cui, Y. H. Pang and H. Qian, Electrochim. Acta, 2012, 59, 91.

179 A. Martin, J. Hernandez-Ferrer, L. Vazquez, M. Martinezb and A. Escarpa, RSC Adv., 2014, 4, 132.

180 Y. Ye, L. Gan, L. Dai, H. Meng, F. Wei, Y. Dai, Z. J. Shi, B. Yu, X. F. Guo and G. G. Qin, J. Mater. Chem., 2011, 21, 11760.

181 B. Chitara, L. S. Panchakarla, S. B. Krupanidhi and C. N. R. Rao, Adv. Mater., 2011, 23, 5419.

182 E. U. Stützel, T. Dufaux, A. Sagar, S. Rauschenbach, K. Balasubramanian, M. Burghard and K. Kern, Appl. Phys. Lett., 2013, 102, 043106.

183 X. Wang, L. Zhi and K. Müllen, Nano Lett., 2008, 8, 323.

184 J. Wu, H. A. Becerril, Z. Bao, Z. Liu, Y. Chen and P. Peumans, Appl. Phys. Lett., 2008, 92, 263302.

185 L. Ju, B. Geng, J. Horng, C. Girit, M. Martin, Z. Hao, H. A. Bechtel, X. Liang, A. Zettl, Y. R. Shen and F. Wang, Nat. Nanotechnol., 2011, 6, 630.

186 M. Freitag, T. Low, W. Zhu, H. Yan, F. Xia and P. Avouris, Nat. Commun., 2013, 4, 1951. 